\title{
Structured Overlapping Grid Simulations of Contra-Rotating Open Rotor Noise
}

\author{
Jeffrey A. Housman*1 and Cetin C. Kiris ${ }^{\dagger 1}$ \\ ${ }^{1}$ NASA Ames Research Center, M/S 258-2, Moffett Field, CA 94035
}

\begin{abstract}
Computational simulations using structured overlapping grids with the Launch Ascent and Vehicle Aerodynamics (LAVA) solver framework are presented for predicting tonal noise generated by a contra-rotating open rotor (CROR) propulsion system. A coupled Computational Fluid Dynamics (CFD) and Computational AeroAcoustics (CAA) numerical approach is applied. Three-dimensional time-accurate hybrid Reynolds Averaged Navier-Stokes/Large Eddy Simulation (RANS/LES) CFD simulations are performed in the inertial frame, including dynamic moving grids, using a higher-order accurate finitedifference discretization on structured overlapping grids. A higher-order accurate freestream preserving metric discretization with discrete enforcement of the Geometric Conservation Law (GCL) on moving curvilinear grids is used to create an accurate, efficient, and stable numerical scheme. The aeroacoustic analysis is based on a permeable surface Ffowcs Williams-Hawkings (FW-H) approach, evaluated in the frequency domain. A timestep sensitivity study was performed using only the forward row of blades to determine an adequate time-step. The numerical approach is validated against existing wind tunnel measurements.
\end{abstract}

\section{Introduction}

Contra-rotating open rotor (CROR) propulsion systems enable a dramatic increase in fuel efficiency over modern turbofan engines. This has renewed interest in CROR concepts, started in the 1980s, to address increasing aviation fuel prices and environmental concerns. The primary challenge of a commercially viable CROR engine for a single-aisle transport aircraft is the reduction of noise emissions. Exclusion of a nacelle to shroud the open rotor blades can increase directional noise by 10 to $30 \mathrm{~dB}$. While propulsion/airframe installation effects can cause non-uniform incoming flow to a coaxial CROR which may increase the radiated noise further. In order to design low-noise open rotor systems, accurate and efficient prediction tools are required. The present study focuses on the validation of high-fidelity prediction tools capable of simulating the complex noise generation mechanisms of CROR systems as well as engine installation effects.

Prediction of noise from a CROR propulsion system has been reported by several researchers. ${ }^{1-4}$ In particular, the prediction of tones generated by the model scale version of the General Electric (GE) F31/A31 blade set at cruise conditions has been reported by Envia. ${ }^{5}$ This blade set contains twelve forward and ten aft blades, and experimental tests have been performed in the NASA 8-foot by 6-foot high-speed wind tunnel. Near-field acoustic wind tunnel measurements and aerodynamic loads on the blades from this high-speed test will be used in the validation of the current proposed simulation methodology. In Envia, ${ }^{5}$ prediction of a subset of the acoustic tones was considered in which a nonlinear harmonic balance approach, implemented in a commercially available software package, was utilized for a single blade passage in combination with a NASA acoustic prediction code. This allowed specific tones associated with linear combinations of the forward and aft blade passing frequencies to be computed directly. The assumption of modeling a single blade passage does not allow angle of attack variations nor installation effects to be considered, and the nonlinear harmonic balance approach is not efficient if broadband content of the radiated noise is not negligible (as

\footnotetext{
*Research Scientist, Computational Aerosciences Branch, NAS Division, AIAA Senior Member

${ }^{\dagger}$ Branch Chief, Computational Aerosciences Branch, NAS Division, AIAA Senior Member

This material is declared a work of the U.S. Government and is not subject to copyright protection in the United States.2016
} 
may be the case in certain azimuthal directions). Instead, the current approach follows ${ }^{6-8}$ in which the full-annulus of the forward and aft blade rows along with the hub are modeled in a three-dimensional timeaccurate moving grid simulation. To extend the current state-of-the-art in CROR simulations, higher-order accurate finite-difference discretizations and hybrid RANS/LES turbulence modelling are used to increase the modeling fidelity compared to existing second-order accurate unsteady RANS simulations reported in the literature. This enables a highly accurate simulation strategy which can be used to assess both aerodynamic and aeroacoustic aspects of CROR designs and their installation into a full aircraft.

In Section II the geometry of the open-rotor configuration is specified along with the conditions of the test case. Next, the computational methodology is described including the higher-order accurate discretization and the modifications to the hybrid Reynolds Averaged Navier-Stokes/Large Eddy Simulation (RANS/LES) turbulence model. Details of the structured overlapping grid system and permeable acoustic surface used in the analysis are presented in Section III.C. The computed results, including comparisons to experimental data and a time-step sensitivity study, are shown in Section IV. Finally, the work is summarized in Section V.

\section{Geometry and Flow Conditions}

The CROR configuration used in the present study consists of the GE F31/A31 blade set mounted on a hub installed in the NASA 8-foot by 6-foot high-speed wind tunnel, see Figure 1 (a). The forward blade row contains twelve blades with a tip diameter of $0.66 \mathrm{~m}$ and a pitch angle of 64.4 degrees. The aft blade row contains ten blades with a slightly smaller tip diameter of $0.63 \mathrm{~m}$ to help reduce impingement of the forward rotor blades tip vortices onto the aft blades, and a pitch angle of 61.8 degrees. Acoustic measurements are gathered from 17 flush mounted Kulites on a flat plate which is positioned over the hub at five different vertical distances, as shown in Figure 1 (b). Figure 2 shows an isometric view of the blades and hub along with an inset showing a side view. The forward blade row is colored in blue and rotates clock-wise with respect to the downstream flow direction, along with the orange section of the hub. The aft blade row is colored red and rotates counter-clockwise along with the purple section of the hub. The nose and downstream sections of the hub are shown in gray. The inflow Mach number of the tunnel is set to 0.78 to emulate cruise conditions, and the present simulations are performed at zero degrees angle of attack. Both the forward and aft rotors are run at $6848 \mathrm{RPMs}$ resulting in a tip Mach number of 1.04 and 1.03 for the forward and aft blades respectively. Measured thrust on the individual blade rows will be used to assess the prediction of the aerodynamic loads. To validate the acoustic predictions, measurements from the seventeen Kulites at several vertical heights are used for comparison. The sampling rate of the Kulites used in the experiment was 200 $\mathrm{kHz}$ over a 15 second time interval. The spectra with a frequency band-width of $12.2 \mathrm{~Hz}$ were computed from the time series and used for validation in the current study.

\section{Computational Methodology}

The Launch Ascent and Vehicle Aerodynamics (LAVA) solver framework ${ }^{9}$ is utilized for the computational study. LAVA offers highly flexible meshing options and was developed with the intent of modeling highly complex geometry and flow fields. The framework supports Cartesian and curvilinear structured grids as well as unstructured arbitrary polyhedral meshes. Overset grid technology ${ }^{10}$ is used to couple the solutions across the different overlapping meshes. In this study, the curvilinear structured overlapping grid methodology is applied. The advantage of this approach for viscous aeroacoustic analysis of CROR applications includes the ease at which highly anisotropic grids can be generated to capture boundary layers, wakes from the blades, and tip vortices, the high efficiency and low memory footprint of the corresponding numerical algorithm, the straightforward and inexpensive extension to higher-order low-dissipation convective flux discretizations which are necessary for capturing flow/acoustic coupling, and the relative motion capabilities inherent in the overset grid methodology. Allowing the structured grids to overlap each other not only simplifies the process of generating the grids (in comparison to structured multi-block patched grids), it also allows for high quality (less-skewed) meshes and a modular approach to adding both geometric components and anisotropic meshes for resolving important flow features. Best practices on overset mesh generation ${ }^{11}$ are expanded upon for aeroacoustic CROR applications in this work. The Chimera Grid Tools (CGT) ${ }^{12}$ software package is used to generate the grids, while hole cutting and connectivity for the overset grid system is performed using the DCF ${ }^{13,14}$ routines in OVERFLOW. ${ }^{15}$ If viscous effects are negligible, aeroacoustic analysis can be performed using the Cartesian grid immersed boundary methodology in LAVA. ${ }^{16}$ The immersed boundary approach is 
explored for open rotor tonal noise prediction for the same configuration in a companion paper, ${ }^{17}$ at both high-speed and low-speed conditions. In addition, the low-speed configuration is also being analyzed using two additional NASA developed CFD codes OVERFLOW and FUN3D. ${ }^{18}$

The compressible hybrid RANS/LES equations are solved using a finite-difference formulation applied to the curvilinear transformed system of equations in strong conservation law form. ${ }^{19}$ The Spalart-Allmaras $(\mathrm{SA})^{20}$ turbulence model is used as the base RANS closure model, and a modified version of the Detached Eddy Simulation (DES) model ${ }^{21,22}$ (modifications described in III.B) is used to transition from RANS to LES with a Smagorinsky-like sub-grid scale model in the LES regions. Implicit second-order backward differencing is used in physical time and the discretized equations are marched in pseudo-time to a converged solution for each physical time-step. The nonlinear system of equations at each time-step are linearized and an alternating line-Jacobi relaxation procedure is applied. Local pseudo time-stepping is used to accelerate convergence. Domain decomposition and the Message Passing Interface (MPI) are used to enable a scalable parallel algorithm.

Resolution of acoustic waves to the far-field using high-fidelity CFD is still not feasible because of the large number of grid points necessary to resolve the highest frequencies of interest and the long timeintegration necessary to capture the lowest frequencies. Even at 5 points-per-wavelength (PPW) resolution capacity, the volume mesh will be several billion grid points. Alternatively, linear acoustic propagation models such as Ffowcs Williams-Hawkings $(\mathrm{FW}-\mathrm{H})^{23}$ are accurate, efficient, and only rely on adequate discretization of a two-dimensional surface embedded in a three-dimensional space (when the volumetric source terms are excluded from the model). Both permeable and non-permeable frequency domain $\mathrm{FW}-\mathrm{H}$ formulations are implemented in LAVA, which closely follows the work of Lockard, ${ }^{24}$ but utilizes unstructured surface triangulations embedded within the structured overlapping grid system instead of structured grid subsets. This allows the same acoustic propagation procedure to be used for the Cartesian, curvilinear, and unstructured polyhedral grid types supported in LAVA. No corrections to remove hydrodynamic fluctuations from the time-accurate solution variables recorded on the permeable surface have been implemented in the present approach, this may lead to some over-prediction of the far-field noise levels.

\section{III.A. Higher-Order Finite-Difference Method}

Higher-order accurate finite-difference schemes have been shown to be an effective strategy for accurately and efficiently capturing noise generation phenomenon using LAVA. ${ }^{25}$ A thorough study comparing several higher-order finite-difference methods on Cartesian grids (within the LAVA framework) was reported recently. ${ }^{26}$ Results from this study indicated that higher-order Weighted Essentially Non-oscillatory (WENO) schemes $^{27}$ performed well in both resolution (PPW), shock capturing, and robustness to harsh flow conditions. A natural extension of finite-difference WENO schemes to curvilinear grids are the higher-order Weighted Compact Nonlinear Schemes (WCNS). ${ }^{28}$ The WCNS method consists of WENO interpolation (as opposed to reconstruction) of left and right states to the half grid points, followed by evaluation of the numerical flux at the half points by an approximate (or exact) Riemann solver or flux vector splitting scheme, and concluding with a higher-order central finite-difference operator at the grid points which depends on the numerical fluxes at the half points in either an implicit (i.e. compact) or explicit form. Standard WENO finite-difference methods do not satisfy the Geometric Conservation Law (GCL) when applied to curvilinear equations in strong conservation law form on non-Cartesian grids. Instead, WENO interpolation can be combined with higher-order central-difference operators resulting in a free-stream preserving (i.e. GCL satisfying) numerical discretization provided that identical central differencing operators are used for discretizing the metric terms as well as the convective (and outer derivative of the viscous) fluxes. ${ }^{29,30}$ An additional advantage of WCNS over WENO is the ability to use approximate Riemann solvers. Standard finite-difference WENO methods require the use of flux vector splitting methods for numerical flux evaluation. In this work, a modified version of the Roe numerical flux is used. ${ }^{31-33}$

A side-effect of using higher-order central difference operators applied to numerical fluxes at the half grid points, which depend on higher-order WENO interpolation, is the much wider stencil required for the same order of accuracy compared to the standard finite-difference WENO method. To remove this dependency, higher-order central difference operators using a combination of the numerical fluxes at the half grid points and the physical fluxes at the grid points have been developed. ${ }^{34,35}$ This approach allows for up to fifth-order accuracy using a seven-point stencil by combining fifth-order WENO interpolation with a sixth-order hybrid central difference operator which is used in the present analysis. In addition to upwind biased WENO interpolation, a blending of central based interpolation is used to increase the maximum 
frequency resolution of the scheme. The blending factor between central and upwind biased interpolation is based on the local Mach number at the half grid point. ${ }^{36}$ In order to preserve the discrete enforcement of the GCL on moving curvilinear grids, the method developed in Thomas and Lombard ${ }^{37}$ and its extension to the second-order backward differencing scheme in time ${ }^{38}$ have been implemented into the LAVA structured overset grid solver. This approach is not only free-stream preserving for rotating grid systems, but also for time-dependent volume deforming grids which will allow the method to be applicable to model aero-elastic effects of the blades in the future.

\section{III.B. Modifications to Hybrid RANS/LES model}

The Detached Eddy Simulation (DES) ${ }^{21,22}$ and Delayed DES ${ }^{39-41}$ (DDES) turbulence model closures are well-tested hybrid RANS/LES models for highly separated flows. In the original DES model, the transition between RANS and LES models was based strictly on local mesh size relative to the wall-distance. For geometries with a wide range of geometric length scales, such as a rotor blade with finite-thickness leading and trailing edges, the local mesh spacing may become small (but not small enough) to force transition from the RANS model to the LES model in places where the model is not appropriate. This brought about the delay modification to the model denoted DDES which attempts to maintain RANS mode in the attached boundary layer.

One critique of hybrid RANS/LES models is the slow development of three-dimensional turbulent structures even when the spatial and temporal resolution is more than enough to capture them. This is often caused by the definition of the local length scale in the model, which for the DDES model is the largest edge length associated with the cell (or dual cell) of a grid point. An alternative length scale definition developed using the Zonal DES approach ${ }^{42}$ utilizes a normalized vorticity vector together with combinations of two-dimensional length scale estimates. The normalized vorticity vector allows the span-wise direction to be identified and removes the grid length spacing in the span-wise direction (which may be large) from the length scale until three-dimensional structures are formed. At this point, the normalized vorticity vector is not aligned with any particular mesh direction and the standard length scale is recovered. Utilization of the normalized vorticity vector along with a more conservative estimate of the mesh spacing has been developed $^{43}$ and is used in the present work.

\section{III.C. Structured Overlapping Grid System and Permeable Acoustic Surface}

A script for the structured overlapping grid system of the GE F31/A31 CROR model was developed in less than two days using the CGT ${ }^{12}$ software package. Several mesh resolutions for the grid system have been generated, but only results for the coarse grid system are presented in Section IV. The coarse grid system consists of 123 zones and 164.6 million grid points, with triple fringe layers to accommodate higher-order finite-difference discretizations. Fringe points which could not find valid donor cells for solution interpolation, called orphan points, have been eliminated for the entire grid system throughout the blade rotations by ensuring proper mesh overlap. All grids were generated using CGT v2.1p. The volume grids are first generated in the default position, then the grid and connectivity are precomputed for each time-step of a full rotation before the flow solver is applied. Each instance (i.e. time-step) of the grid and connectivity requires seven minutes to compute and store, but this can be performed independently. Scripts were developed to submit each instance in parallel using the Pleiades Supercomputer at NASA Ames Research Center which reduced the pre-processing wall-clock time to only a few hours. The computed wall $y^{+}$is between one-half and one on the hub, but increases to approximately five at the blade tips. The surface grid for each blade consists of an O-grid wrapping around the main body of the blade, a collar grid which is hyperbolically marched onto the hub, and a cap grid covering the blade tip. Images of the forward blade surface grid are shown in Figure 3. By utilizing the CGT scripting library, a single blade grid is generated for the forward and aft rows and the remaining blades are constructed by rotating the single blades. The collar grids are constructed on the fly in the script allowing the blade angle to be parameterized for future configurations. The hub surface grid is generated with one degree spacing in the circumferential direction and clustered in the stream-wise direction near the collar grids of the blades for proper overset communication. Once the surface grids are complete, volume grids are generated by hyperbolically marching in the wall normal direction with tight clustering near the wall. Images of the grid system are shown in Figure 4.

For acoustic propagation, a permeable surface is embedded within the structured overlapping grid system and tri-linear interpolation is used to record the unsteady flow data onto the face centers of the surface 
triangulation. In this simulation, three permeable acoustic surfaces were generated for the propagation, shown in Figure 6. Surface 1 consists of a tight fitting cylinder which encloses the forward and aft rotors but excludes the wake. Surface 2 encloses the front end of the hub and the downstream portion of the wake where the CFD grid is still relatively well resolved. Surface 3 contains the same front portion of the cylinder as surface 2 but extends much further downstream stopping at the straight section of the hub. The CFD mesh coarsens rapidly before the closure of the surface 3 which affects the predicted acoustic radiation from the rotor wake. Each of the permeable surfaces used has the same diameter of $0.4957 \mathrm{~m}$ and the triangle edge length is approximately $0.01 \mathrm{~m}$. Sensitivity of the predicted far-field noise to the different acoustic surfaces is examined in Section IV.

A finer grid system was also generated by refining the stream-wise spacing on the blades, reducing the circumferential spacing on the hub to half-a-degree, increasing the resolution of the wake, reducing the wall spacing to maintain $y^{+} \approx 1$ at the blade tips, and extending the far-field boundary distance. The finer grid consists of 303 zones and 427.9 million grid points still maintaining triple fringe throughout the grid system with zero orphan points.

In order to determine an appropriate temporal resolution, a time-step study was performed on a simplified grid system generated from a sub-set of the finer grid. The grid system consists of a one-twelfth single passage cylindrical hub and a single forward blade. Figures 5 (a)-(d) show an isometric view of the simplified hub with the forward blade surface grid, a constant $y=0$ slice of the hub grid showing the wake resolution in the stream-wise and radial directions, an isometric view of stream-wise grid slices of the hub and forward blade showing the circumferential and radial spacing, and a zoomed out view of the entire grid system with the model highlighted by a small magenta circle located in the lower middle of the plot. Simulations of the simplified model can be computed at significantly less expense since only one-twelfth of the geometry is modeled, and the entire grid system is rotated in a single direction eliminating the need to recompute the connectivity at each time-step.

\section{Computational Results}

A qualitative characterization of the flow-field, based on the coarse grid simulation using a time-step of 0.5 degrees rotation-per-step, is first described. Figure 7 plots the instantaneous (a) pressure and (b) magnitude of the density gradient on a slice through the centerline of the hub. A section of the cutting plane is absent from the image since this section is in relative motion. The pressure field shows the formation of shocks traveling upstream from the rotors caused by the displacement of the air by the blade tips which are traveling supersonically. A weak shock just downstream of the aft blade row and a strong fish-tail shock downstream of the final turn in the hub before the long straight section is observed. The magnitude of the density gradient shows a clear representation of the tip vortices convecting downstream and interacting with the fish-tail shock to form an additional acoustic noise generator. Additionally a radiating acoustic wave pattern is observed emanating from the intersection of the aft blades and the hub and propagating at an angle of approximately 40 degrees relative to the hub centerline. Figure 8 (a)-(c) plots the pressure on stream-wise slices through the forward rotor, between the two rotors, and the aft rotor, respectively. The wave pattern through the forward blade set is dominated by the clockwise rotation of the forward rotor near the blades with an interaction with the aft rotor observed at approximately two tip radii from the hub centerline. In contrast, the wave pattern between the blades shows a very strong interaction between the waves generated by both the forward and aft rotors, as expected. The aft rotor shows wave patterns strongly associated with the counter-clockwise rotation of the aft blades near the hub. The interaction of the wake from the forward rotor with that of the aft rotor is illustrated in Figure 9 which plots an iso-contour of the vorticity magnitude colored my the local Mach number. Tip vortices from the forward blades travel downstream toward the aft rotor and impact the tip of the aft blades. These vortices then travel just below the tip vortices generated by the aft blade row. This interaction of the tip vortices continues in the wake of the rotors before interacting with the fish-tail shock.

\section{IV.A. Aerodynamic Loads Comparison}

For a more quantitative assessment of the numerical simulation, the aerodynamic loads are compared to the experimental data in Figure 10. The plot shows the combined thrust generated by the forward and aft rotor blades. In Figure 10 (a) the evolution of the thrust is plotted showing that approximately 2 rotor revolutions 
are required to remove the initial transients from the impulsive start. The thrust then appears to converge in approximately $4-6$ rotor revolutions with the exception of a low frequency amplitude oscillation. This oscillation is associated with a low frequency mode coupling between the outer boundary and the aft rotor, which was identified after the simulation was complete by analyzing the perturbation pressure. The mode was generated because the outer boundary was located too close to the model at only 15 tip radii from the hub centerline. For the finer grid, the outer boundary extended over 100 tip radii from the hub centerline which removed this behavior, as will be demonstrated in the time-step sensitivity study. A comparison of the predicted thrust over the final four rotor revolutions to the experimental data is shown in Figure 10 (b). The time-average of the thrust is $3.9 \mathrm{kN}$ which is 5 percent larger than the measured thrust in the experiment. The over-prediction is likely caused by the large $y^{+}$values of the coarse grid at the rotor tips and should improve on the finer mesh.

\section{IV.B. Aeroacoustic Tones Comparison}

The sound pressure level (SPL) spectrum shown in Figure 11 was generated by dividing the time-series over $3-11$ rotor revolutions into 5 overlapping sections, each section includes a duration of 4 rotor revolutions, with an overlap of 3 rotor revolutions. Then the Fast Fourier Transform (FFT) is applied to each of the 5 sections independently and the $\mathrm{FW}-\mathrm{H}$ solver is used to propagate each section to the observer (Kulite) locations. The spectrum generated by each section is then averaged to form the final SPL spectrum. Figure 11 (a) plots the predicted SPL spectrum at Kulite 9, located $0.51 \mathrm{~m}$ above the hub centerline directly above the aft rotor, versus the wind tunnel data. The wind tunnel data has been corrected by a $6 \mathrm{~dB}$ subtraction to account for the reflection of the acoustic waves by the flat plate fixed above the model. Both the pure CFD and the coupled CFD/CAA results are shown to illustrate the quality of the coupled approach at a location in which the CFD mesh is still relatively fine. The three distinct permeable acoustic surfaces with different fore-body and wake extents, described in Section III.C, are included in the analysis. The tones associated with the first and second individual Blade Passing Frequencies (BPFs) are well-predicted up to and above $4 \mathrm{BPF}_{1}$ and $4 \mathrm{BPF}_{2}$. The predicted SPL levels of the interacting tones associated with sums of the two blade passing frequencies up to $\mathrm{BPF}_{1}+3 \mathrm{BPF}_{2}$ match well, but the amplitude at $2 \mathrm{BPF}_{1}+2 \mathrm{BPF}_{2}$ and beyond is too low. The tones associated with differences of the two blade passing frequencies are considerably lower than the measured data in the wind tunnel. Theoretically the difference tones do not radiate as efficiently as the sum tones, and this is observed in the CFD/CAA prediction. The larger amplitude difference tones observed in the wind tunnel data are likely caused by imperfections in both blade shapes and the installation into the hub. As explained in Envia, ${ }^{5}$ the installed blade angles vary by as much as a tenth of a degree between blades on the same rotor, which can cause the increased SPL at say $2 \mathrm{BPF}_{1}-\mathrm{BPF}_{2}$. Figure 11 (b) plots the SPL spectrum for the same Kulite but at the further distance of $1.16 \mathrm{~m}$ from the hub centerline. Similar to the results at $0.51 \mathrm{~m}$, the SPL levels of the individual blade passing frequencies are well captured. A noticeable difference is observed in the SPL prediction between the different acoustic surfaces at $\mathrm{BPF}_{1}+2 \mathrm{BPF}_{2}$ and $3 \mathrm{BPF}_{1}+\mathrm{BPF}_{2}$. At both frequencies, the second acoustic surface appears to generate the best prediction. This surface extends into the wake of the blades but not so far downstream that the mesh coarsening effects play a dominant role.

In addition to the narrow-band spectrum, Figure 12 compares the SPL for the dominant tones as a function of the vertical distance from the hub centerline between the computation and experiment. Based on the comparison of the narrow-band spectrum, permeable surface 2 was chosen for the FW-H acoustic propagation of the dominant tones. Kulites 8 and 9 show similar trends. The SPL of $\mathrm{BPF}_{1}$ is predicted within $1 \mathrm{~dB}$ initially, then shows an over-prediction of less than or equal to $5 \mathrm{~dB}$ as the vertical distance increases. For $\mathrm{BPF}_{2}$ the $\mathrm{SPL}$ is over-predicted by a little less than $5 \mathrm{~dB}$ over most of the vertical distance, but has an almost identical match to the experiment at the furthest vertical distance. The first sum tone $\mathrm{BPF}_{1}+\mathrm{BPF}_{2}$ is predicted within $5 \mathrm{~dB}$ over the vertical distance except at Kulite 8 vertical distance $0.87 \mathrm{~m}$. At this location the computation grossly under-predicts the SPL by almost $8 \mathrm{~dB}$. This does not appear to be the case at any of the other vertical distance locations and must be investigated further for a better understanding of the difference. Excluding this single point, the prediction is within $5 \mathrm{~dB}$ for the dominant tones over the vertical distance and appears qualitatively similar to the measure data. Spectral analysis was also performed on a $y=0$ cutting plane through the hub to get a better understanding of the locality of the dominant tones. Figure 13 shows a frequency diagram plotting the frequency at which the largest amplitude in the narrow-band spectrum is obtained for each spatial location on the cutting plane. The gold region represents $\mathrm{BPF}_{1}$ and is observed near the forward rotor blades as expected. $\mathrm{BPF}_{2}$ is shown in teal and not 
only dominates the aft blade region but appears to encompass much of the upstream and radial portion of the domain. The first two sum tones, $\mathrm{BPF}_{1}+\mathrm{BPF}_{2}$ and $\mathrm{BPF}_{1}+2 \mathrm{BPF}_{2}$ are shown in red and highlight the wake region where the tip vortices and wakes from the forward and aft blade rows interact with each as observed in Figure 9. The purple region in Figure 13 indicates where the computational mesh resolution was not fine enough to resolve the acoustic wave propagation. The permeable surface should be sized close enough to the body to exclude this region, but far enough to reduce the parasitic effects of the hydrodynamic fluctuations. Surface 2 is a good compromise in this respect. A more detailed explanation of the spatial locality dependence of the dominant tones is shown in Figures 14-16 which plot the amplitude and phase for the Fourier coefficients on the $y=0$ cutting plane through the hub for $\mathrm{BPF}_{1}, \mathrm{BPF}_{2}$, and $\mathrm{BPF}_{1}+\mathrm{BPF}_{2}$, respectively. Figure 14 (a) shows that $\mathrm{BPF}_{1}$ generates large amplitudes near the forward rotor and the tip vortices which interact with the fish tail shock. The phase plot indicates acoustic radiation patterns from both the forward rotor and fish tail shock regions. Figure 15 (a) shows that $\mathrm{BPF}_{2}$ attains large amplitudes near the aft rotor, along the tip vortex, across the weak shock just downstream of the aft rotor, and at the fish tail shock. The phase plot in Figure 15 (b) indicates a strong forward radiation pattern upstream of the forward rotor along the nose of the hub. A similar radiation pattern as $\mathrm{BPF}_{1}$ is observed near the fish tail shock region. Figure 16 (a) plots the amplitude associated with $\mathrm{BPF}_{1}+\mathrm{BPF}_{2}$ showing large values in the tip vortex and wake regions. The phase plot in Figure 16 (b) shows a strong radiation pattern emanating from the wake in the 45 degree direction from the hub centerline.

\section{IV.C. Time-Step Sensitivity Study}

Before the finer mesh simulation is performed, a thorough time-step sensitivity study was completed to determine the appropriate physical time-step. With the use of higher-order accurate spatial discretizations, the temporal accuracy of the second-order backward differencing in time must be assessed since this will be the leading source of truncation error as the mesh is refined. As described in Section III.C, a structured overlapping grid system of a simplified version of the hub geometry corresponding to a single passage of the forward rotor, i.e. one-twelfth of the full annulus, was created from the finer grid of the full geometry. This was used in the time-step sensitivity study in which unsteady simulations were performed at timesteps associated with $0.5,0.25,0.125,0.0625,0.03125$ degrees rotation-per-step. From these simulations the resolution of the three-dimensional vortex breakdown and capturing of the pressure wave perturbations were compared to determine an efficient and accurate time-step. For each simulation, the pseudo-time subiteration procedure was performed for 20 iterations or 2-orders of magnitude residual reduction, whichever occurred first. Figure 17 (a)-(e) shows contour plots of instantaneous vorticity magnitude on a $y=0$ cutting plane through the hub. The tip vortex and wake from the blade are well captured even with the coarsest time-step. The large vorticity content interacting with the hub downstream of the blade is generated by a separated flow region where the blade intersects the hub near the trailing-edge. This separated flow region generates vorticity which travels downstream and interacts with the boundary layer on the hub as illustrated in Figure 17 (f). The highly unsteady flow may cause a broadband component to the acoustic spectrum. Examining Figure 17 (a) more closely, the structures appear excessively smoothed out and some three-dimensional content is missing compared to the images from the smaller time-steps. Figure 17 (b) shows more structure with the 0.25 degree time-step and retains most of the three-dimensional structures. As the time-step is dropped further the temporal discretization error falls bellow the spatial discretization error and Figures 17 (c)-(e) appear to have identical three-dimensional content and structure. Examining the non-dimension gauge pressure on a radial grid slice above the blade in Figures 18 (a)-(e) shows again that the 0.5 degree time-step is too large to capture all of the acoustic wave features. The 0.25 time-step improves the resolution, while the 0.125 degree time-step appears to contain identical features as those captured using 0.0625 and 0.03125 degree time-steps. In terms of aerodynamic loads, Figure 19 plots the time-history of thrust on a single forward blade for each of the time-steps over 3 to 4 rotor revolutions. Once the initial transients have passed the thrust appears to be invariant to the time-step used at slightly over 3 rotor revolutions. It is also observed that the extended outer-domain boundary has completely removed the low-frequency coupling mode and no oscillations are retained in the time-history of converged thrust. This study suggests that a maximum time-step of 0.25 degrees may be used with the finer grid, but the 0.125 degree time-step appears optimal with respect to efficiency and accuracy of capturing vortex structures and pressure perturbations. Both of these time-steps will be utilized in a future simulation using the finer grid, and results from these computations will be reported in subsequent papers. 


\section{Summary}

The Launch Ascent and Vehicle Aerodynamics (LAVA) solver framework, using structured overlapping grids and a coupled CFD/CAA approach, has been successfully applied to the prediction of tonal noise generated from a model scale contra-rotating open rotor propulsion system. Higher-order accurate spatial discretizations and improved hybrid RANS/LES turbulence modeling were used to improve the state-of-theart for open rotor noise calculations. Results from a coarse grid calculation achieve predicted thrust values within 5 percent and excellent agreement in SPL at the individual Blade Passing Frequencies. SPL predictions of the interaction tones associated with the sum of the two Blade Passing Frequencies are well-captured up to $\mathrm{BPF}_{1}+3 \mathrm{BPF}_{2}$ but quickly degrade at high frequencies. Difference tones are grossly under-predicted compared to experimental data, but these differences are attributed to inaccurate blade installation effects. The prediction of the dominant tones with respect to vertical distance are captured within $5 \mathrm{~dB}$ above the forward and aft blades.

A detailed spectral analysis was performed on a cutting plane through the hub to identify the regions of influence associated with the dominant tones. Blade Pass Frequency 1 mostly influences the forward blade region as expected, while Blade Pass Frequency 2 affects the aft blade region, the upstream portion of the hub, and a large portion of the domain above the two counter-rotating blade rows. The first two sum interaction tones dominate the wake region and generate an acoustic radiation pattern at 45 degrees with respect to the hub centerline. A finer overset mesh was generated and a thorough time-step sensitivity study was performed on a subset of the finer grid. Vortical structures and perturbation pressures were compared using a sequence of decreasing time-steps. The results indicate that 0.5 degree time-step is too large for the finer grid, while 0.25 degrees may be sufficient. The results from 0.125 degrees appeared indistinguishable from the smaller time-steps and provide an efficient and accurate time-step to be used for the finer grid simulation. Results from finer grid simulations will be reported in a future paper.

\section{Acknowledgements}

This work was supported by the NASA Advanced Air Transport Technology (AATT) project under the Transformative Aeronautics Concepts (TAC) program. The authors would like to thank the members of the experimental test team for access to the wind tunnel data and model geometry. The authors would also like to thank Ed Envia and Chris Miller of NASA Glenn Research Center for many fruitful discussions on modeling open rotor noise and associated meshing requirements, Christoph Brehm of NASA Ames Research Center for creating the freqency diagram and phase/amplitude plots, and the acoustic working group team members at NASA Glenn and NASA Langley research center. Computer time has been provided by the NASA Advanced Supercomputing (NAS) facility at NASA Ames Research Center.

\section{References}

\footnotetext{
${ }^{1}$ Spalart, P., Travin, A., Shur, M., and Strelets, M., "Initial Noise Predictions for Open Rotors Using First Principles," 16th AIAA/CEAS Aeroacoustics Conference, Stockholm, Sweden, June 2010, AIAA-2010-3793.

${ }^{2}$ Rebecca Busch, E., Kebler, M., and Kramer, E., "Aeroacoustics of a High-Fidelity CFD Calculation of a CounterRotating Open Rotor in Take-Off Conditions," 19th AIAA/CEAS Aeroacoustics Conference, Berlin, Germany, May 2013, AIAA-2013-2202.

${ }^{3}$ Ben Nasr, N., Ortun, B., Chelius, A., and Canard-Caruana, S., "Assessment of advanced grid strategies for CFD on open rotor applications," 49th AIAA/ASME/SAE/ASEE Joint Propulsion Conference, San Jose, CA, July 2013, AIAA-2013-3800.

${ }^{4}$ Node-Langlois, T., Wlassow, F., Languille, V., Colin, Y., Caruelle, B., Gill, J., Chen, X., Zhang, X., and Parry, A., "Prediction of Contra-Rotating Open Rotor broadband noise in isolated and installed configurations," 20th AIAA/CEAS Aeroacoustics Conference, Atlanta, Georgia, June 2014, AIAA-2014-2610.

${ }^{5}$ Envia, E., "Contra-Rotating Open Rotor Tone Noise Prediction," 20th AIAA/CEAS Aeroacoustics Conference, Atlanta, Georgia, June 2014, AIAA-2014-2606.

${ }^{6}$ Stuermer, A., "Unsteady CFD Simulations of Contra-Rotating Propeller Propulsion Systems," 44th AIAA/ASME/SAE/ASEE Joint Propulsion Conference, Hartford, Connecticut, July 2008, AIAA-2008-5218.

${ }^{7}$ Stuermer, A. and Yin, J., "Low-Speed Aerodynamics and Aeroacoustics of CROR Propulsion Systems," 15th AIAA/CEAS Aeroacoustics Conference, Miami, Florida, May 2009, AIAA-2009-3134.

${ }^{8}$ Stuermer, A., "Validation of Installation Effect Predictions through Simulations of Contra-Rotating Open Rotors at Low-Speed Flight Conditions," 33rd AIAA Applied Aerodynamics Conference, Dallas, Texas, June 2015, AIAA-2015-2886.

${ }^{9}$ Kiris, C., Barad, M., Housman, J., Sozer, E., Brehm, C., and Moini-Yekta, S., "The LAVA Computational Fluid Dynamics Solver," 52st AIAA Aerospace Sciences Meeting, National Harbor, Maryland, January 13-17, 2014, AIAA-2014-0070.

${ }^{10}$ Steger, J. and Benek, J., "On the Use of Composite Grid Schemes in Computational Aerodynamics," Technical Memorandum 88372, NASA, 1986.
} 
${ }^{11}$ Chan, W., Gomez, R., Rogers, S., and Buning, P., "Best Practices in Overset Grid Generation," 32nd AIAA Fluid Dynamics Conference and Exhibit, St. Louis, Missouri, June 24-26, 2002, AIAA-2002-3191.

${ }^{12}$ Chan, W., "Developments in Strategies and Software Tools for Overset Structured Grid Generation and Connectivity," 20th AIAA Computational Fluid Dynamics Conference, Honolulu, Hawaii, June 2011, AIAA-2011-3051.

${ }^{13}$ Meakin, R. L., "On the Spatial and Temporal Accuracy of Overset Grid Methods for Moving Body Problems," AIAA paper 1994-1925, AIAA 12th Applied Aerodynamics Conference, Colorado Springs, CO, June 1994.

${ }^{14}$ Meakin, R., "The Chimera Method of Simulation for Unsteady Three-dimensional Viscous Flow," Computational Fluid Dynamics Review 1995, edited by M. Hafez and K. Oshima, John Wiley and Sons Ltd, 1995, pp. 70-86.

${ }^{15}$ Jespersen, D., Pulliam, T., and Buning, P., "Recent enhancements to OVERFLOW," 35th Aerospace Sciences Meeting and Exhibit, Reno, NV, January 1997, AIAA 1997-0644.

${ }^{16}$ Brehm, C., Barad, M., and Kiris, C., "An Immersed Boundary Method for Solving the Compressible Navier-Stokes Equations with Fluid-Structure Interaction," 54th AIAA Aerospace Sciences Meeting, San Diego, CA, January 2016.

${ }^{17}$ Brehm, C., Barad, M., and Kiris, C., "Open Rotor Aeroacoustic Analysis with an Immersed Boundary Method," 54th AIAA Aerospace Sciences Meeting, San Diego, CA, January 2016.

${ }^{18}$ Nark, D., Jones, W., Boyd, D., and Zawodny, N., "Isolated Open Rotor Noise Prediction Assessment Using the F31A31 Historical Blade Set," 54th AIAA Aerospace Sciences Meeting, San Diego, CA, January 2016.

${ }^{19}$ Vinokur, M., "Conservation Equations of Gasdynamics in Curvilinear Coordinate Systems," Journal of Computational Physics, Vol. 14, 1974, pp. 105-125.

${ }^{20}$ Spalart, S. and Allmaras, S., "A One-Equation Turbulence Model for Aerodynamic Flows," 30th Aerospace Sciences Meeting and Exhibit, Reno, NV, January 1992, AIAA-92-0439.

${ }^{21}$ Spalart, S. R., Jou, W.-H., Strelets, M., and Allmaras, S. R., "Comments on the feasibility of LES for wings, and on a hybrid RANS/LES approach," First AFOSR international conference on DNS/LES, Ruston, Louisiana, August 1997, Greyden Press.

${ }^{22}$ Strelets, M., "Detached eddy simulation of massively separated flows," 39th Aerospace Sciences Meeting and Exhibit, Reno, Nevada, January 2001, AIAA-2001-0879.

${ }^{23}$ Ffowcs Williams, J. E. and Hawkings, D. L., "Sound generation by turbulence and surfaces in arbitrary motion," Philosophical Transactions of the Royal Society of London A, Vol. 342, 1969, pp. 264-321.

${ }^{24}$ Lockard, D., "A Comparison of Ffowcs Williams-Hawking Solvers for Airframe Noise Applications," 8th AIAA/CEAS Aeroacoustics Conference, Breckenridge, Colorado, June 2002, AIAA-2002-2580.

${ }^{25}$ Brehm, C., Housman, J., and Kiris, C., "Noise Generation Mechanisms for a Supersonic Jet Impinging on an Inclined Plate," Journal of Fluid Mechanics, 2015, In review.

${ }^{26}$ Brehm, C., Barad, M., Housman, J., and Kiris, C., "A Comparison of Higher-Order Shock Capturing Schemes Within the LAVA CFD Solver," 52nd AIAA Aerospace Sciences Meeting, National Harbor, Maryland, January 2014, AIAA-2014-1278.

${ }^{27}$ Shu, C.-W., "High Order Weighted Essentially Nonoscillatory Schemes for Convection Dominated Problems," SIAM Review, Vol. 51, No. 1, 2009, pp. 92-126.

${ }^{28}$ Deng, X. and H., Z., "Developing High-Order Weighted Compact Nonlinear Schemes," Journal of Computational Physics, Vol. 165, 2000, pp. 22-44.

${ }^{29}$ Vinokur, M. and Yee, H., "Extension of Efficient Low Dissipation High Order Schemes for 3-D Curvilinear Moving Grids," Frontiers of Computational Fluid Dynamics, World Scientific, 2002, pp. 129-163, edited by D. A. Caughey and M. M. Hafez.

${ }^{30}$ Deng, X., Mao, M., Tu, G., Liu, H., and Zhang, H., "Geometric conservation law and applications to high-order finite difference schemes with stationary grids," Journal of Computational Physics, Vol. 230, 2011, pp. 1100-1115.

${ }^{31}$ Housman, J., Kiris, C., and Hafez, M., "Preconditioned methods for simulations of low speed compressible flows," Computes and Fluids, Vol. 38, No. 7, August 2009, pp. 1411-1423.

${ }^{32}$ Housman, J., Kiris, C., and Hafez, M., "Time-Derivative Preconditioning Methods for Multicomponent Flows - Part I: Riemann Problems," Journal of Applied Mechanics, Vol. 76, No. 2, February 2009.

${ }^{33}$ Housman, J., Kiris, C., and Hafez, M., "Time-Derivative Preconditioning Methods for Multicomponent Flows - Part II: Two-Dimensional Applications," Journal of Applied Mechanics, Vol. 76, No. 3, March 2009.

${ }^{34}$ Deng, X., Mao, M., Jiang, Y., and Liu, H., "New High-Order Hybrid Cell-Edge and Cell-Node Weighted Compact Nonlinear Schemes," 20th AIAA Computational Fluid Dynamics Conference, Honolulu, Hawaii, June 2011, AIAA-2011-3857.

${ }^{35}$ Nonomura, T. and Fujii, K., "Robust explicit formulation of weighted compact nonlinear scheme," Computers 83 Fluids, Vol. 85, 2013, pp. 8-18.

${ }^{36}$ Thornber, B., Mosedale, A., Drikakis, D., Youngs, D., and Williams, R. J., "An improved reconstruction method for compressible flow with low Mach number features," Theoretical and Computational Fluid Dynamics, Vol. 26, 2012, pp. 523-550.

${ }^{37}$ Thomas, P. and Lombard, C., "Geometric Conservation Law and Its Application to Flow Computations on Moving Grids," AIAA Journal, Vol. 17, No. 10, 1979, pp. 1030-1037.

${ }^{38}$ Nichols, R. and Heikkinen, "Validation of Implicit Algorithms for Unsteady Flows Including Moving and Deforming Grids," 43rd AIAA Aerospace Sciences Meeting, Reno, Nevada, January 2005, AIAA-2005-683.

${ }^{39}$ Spalart, P., Deck, S., Shur, M., Squires, K., Strelets, M., and Travin, A., "A new version of detached-eddy simulation, resistant to ambiguous grid densities," Theoretical and Computational Fluid Dynamics, Vol. 20, May 2006, pp. 181-195.

${ }^{40}$ Shur, M., Spalart, P. R., Strelets, M., and Travin, A., "A hybrid RANS-LES approach with delayed-DES and wallmodelled LES capabilities," International Journal of Heat and Fluid Flow, Vol. 29, 2008, pp. 1638-1649.

${ }^{41}$ Spalart, P. R., "Detached-Eddy Simulation," Annual Review Fluid Mechanics, Vol. 41, 2009, pp. 181-202.

${ }^{42}$ Chauvet, N., Deck, S., and Jacquin, L., "Zonal detached eddy simulation of a controlled propulsive jet," AIAA Journal, Vol. 45, No. 10, 2007, pp. 2458-2473.

${ }^{43}$ Shur, M., Spalart, P. R., Strelets, M., and Travin, A., "An enhanced version of DES with rapid transition from RANS to LES in separated flows," Flow, Turbulence and Combustion, Vol. 95, No. 4, December 2015, pp. 709-737. 


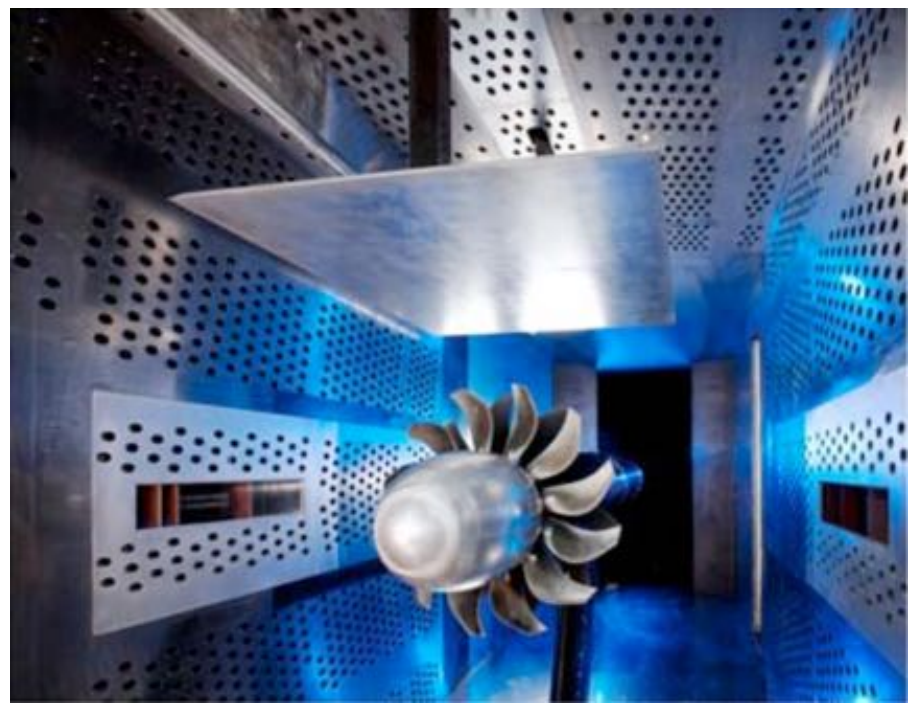

(a)

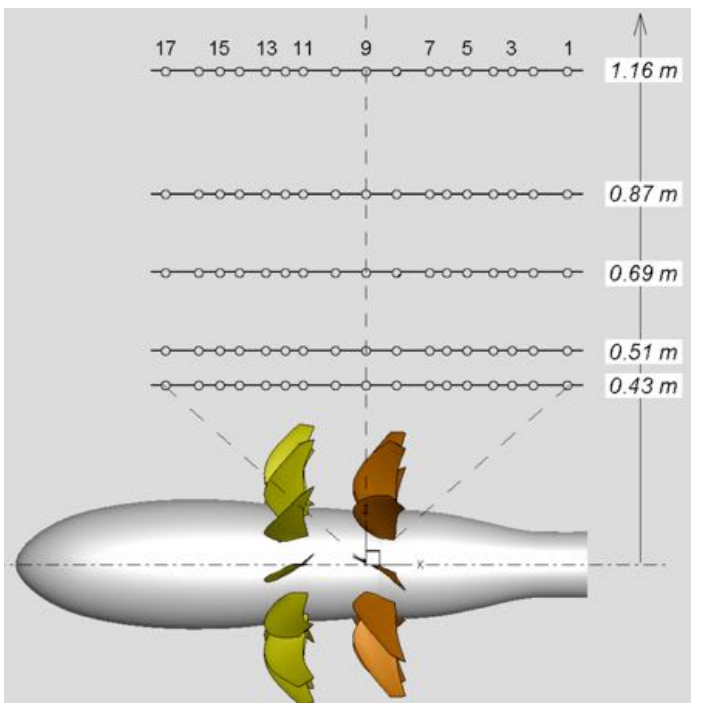

(b)

Figure 1. (a) Scale model of the F31/A31 blade set installed in the NASA 8-foot by 6-foot high-speed wind tunnel. (b) Diagram illustrating the acoustic measurement locations for the wind tunnel experiment. Images reproduced from Envia $^{5}$ pg. 9.

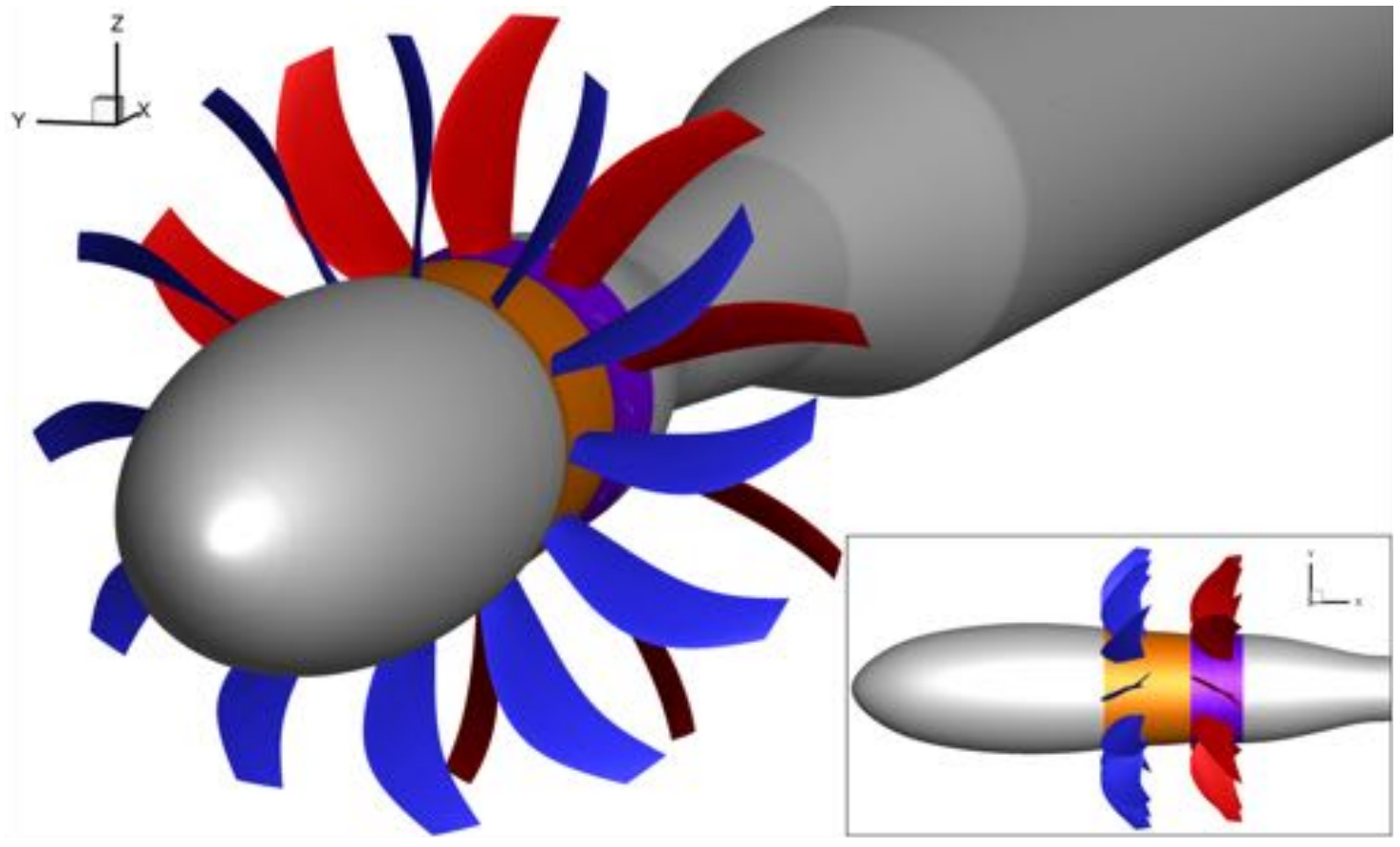

Figure 2. Isometric view of the GE F31/A31 blade set and hub. Inset shows a side view of the blade rows. 


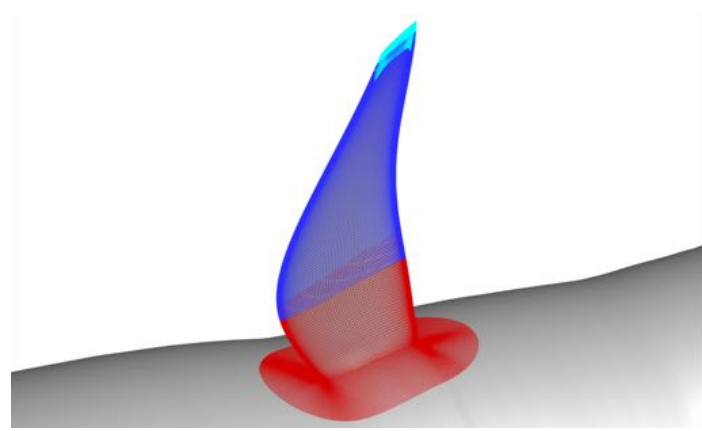

(a)

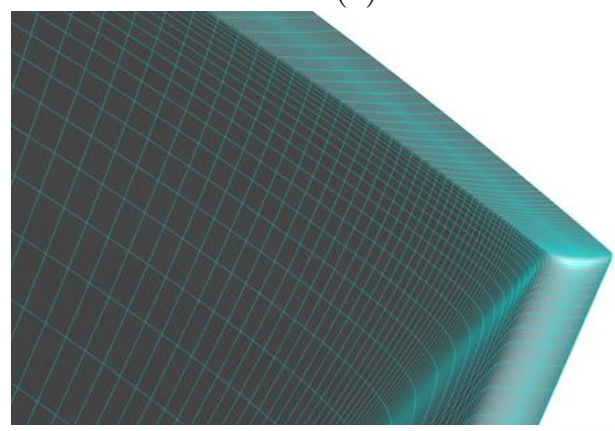

(c)

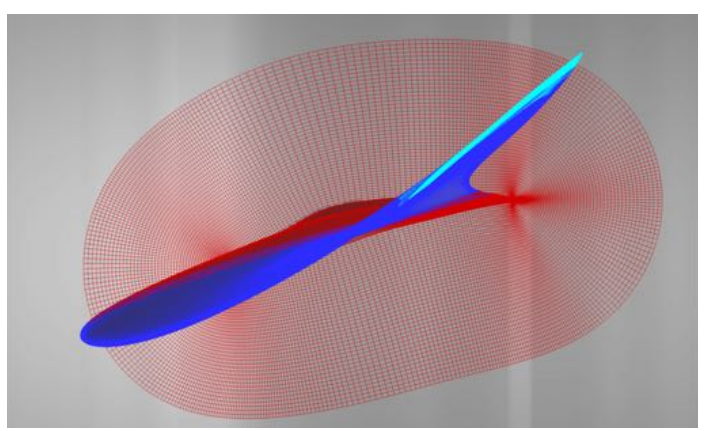

(b)

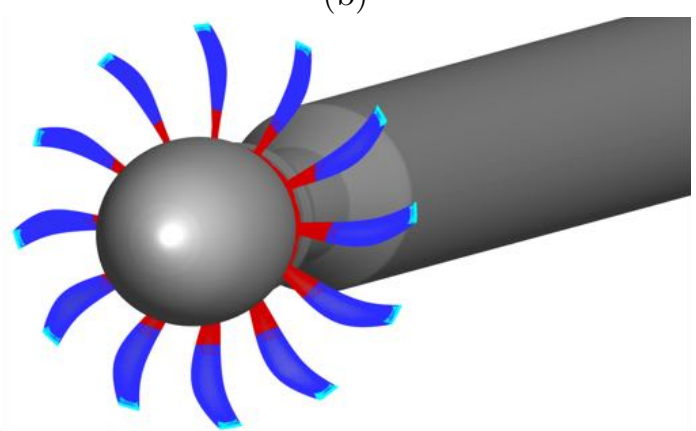

(d)

Figure 3. Surface grid of the forward rotor blade (a) isometric view of a single blade, (b) top-view of the collar grid hyperbolically marched onto the hub surface, (c) close-up view of the finite-thickness trailing-edge of the blade tip, (d) and isometric view of the entire forward blade row illustrating the simple rotation of a single blade to generated the entire blade row.

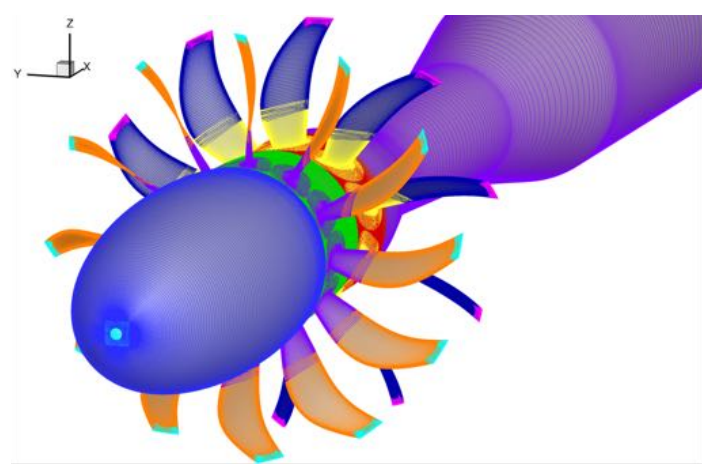

(a)

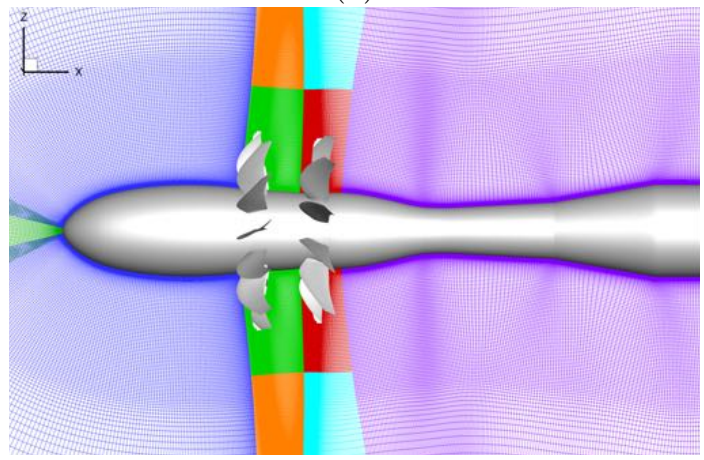

(c)

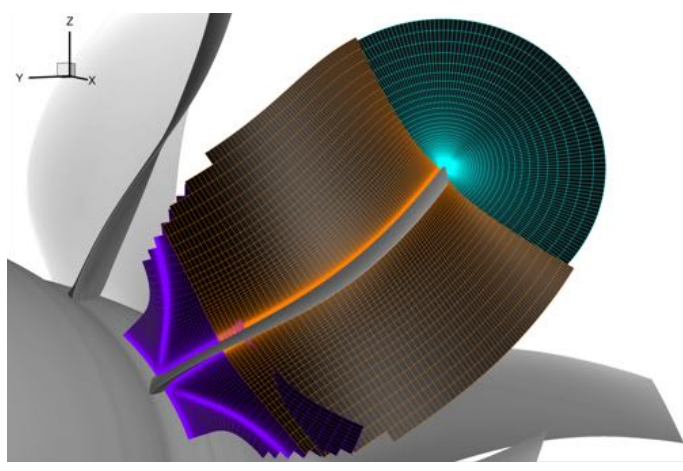

(b)

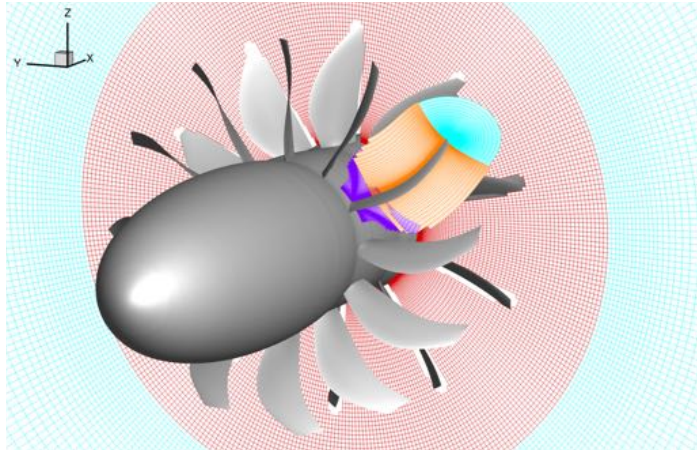

(d)

Figure 4. Several views of the coarse overset grid system (a) isometric view of the surface, (b) slices of the forward blade volume grid, (c) constant $y=0$ cutting plane through the hub, and (d) constant stream-wise slice of the volume grid through the aft rotors along with slices of the forward blade volume grid. 


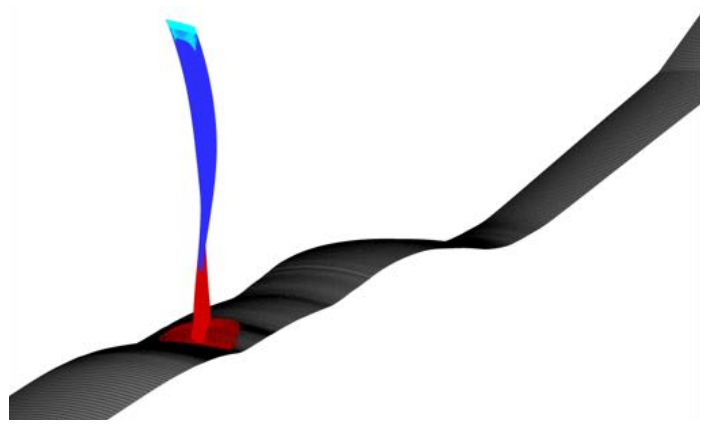

(a)

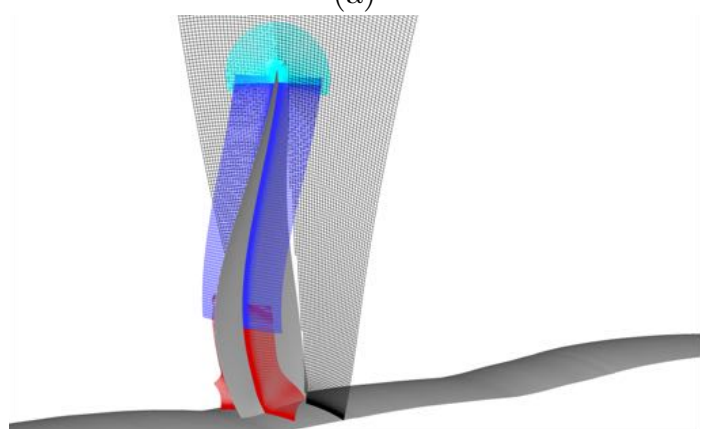

(c)

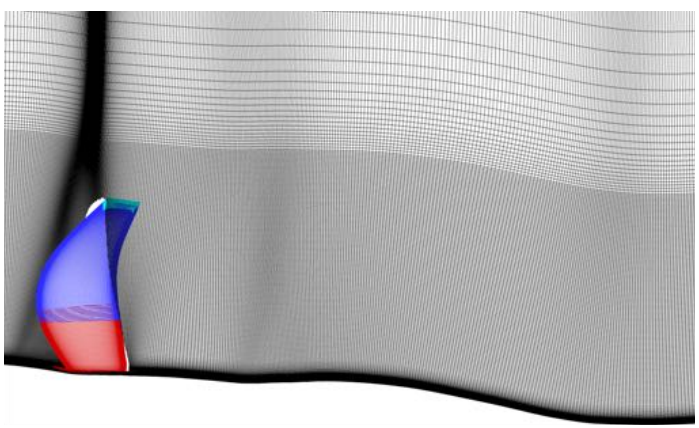

(b)

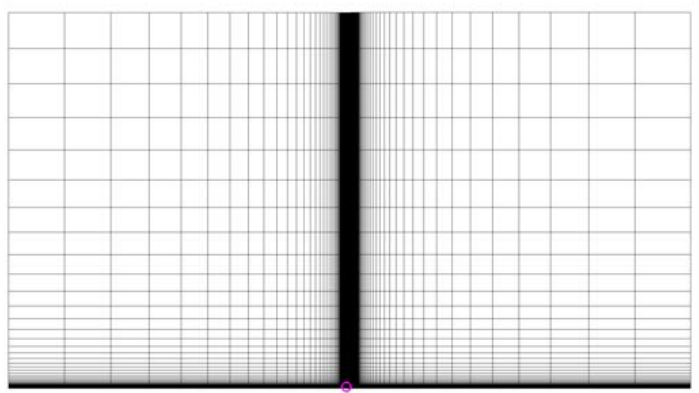

(d)

Figure 5. Structured overset grid system for the medium grid single passage forward rotor time-step sensitivity study showing (a) an isometric view of the simplified hub with with the forward blade surface grid, (b) a constant y = 0 slice of the hub grid showing the wake resolution in the stream-wise and radial direction, (c) an isometric view of stream-wise grid slices of the hub and forward blade showing the circumferential and radial spacing, (d) and a zoomed out view of the entire grid system with the model highlighted by a small magenta circle located in the lower middle of the plot. 


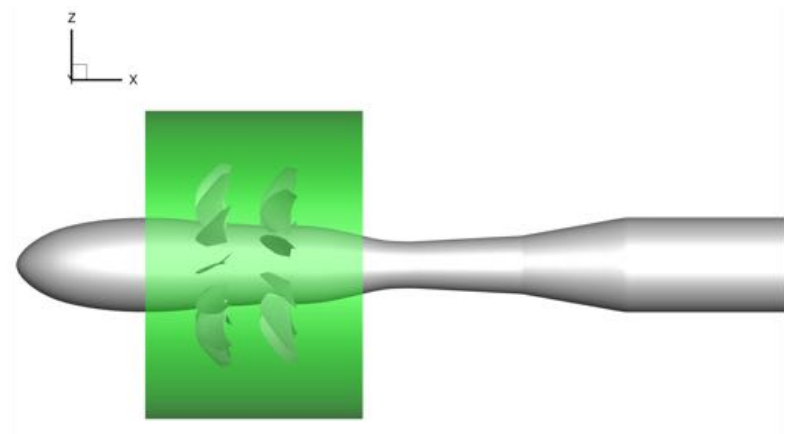

(a) Acoustic Surface 1

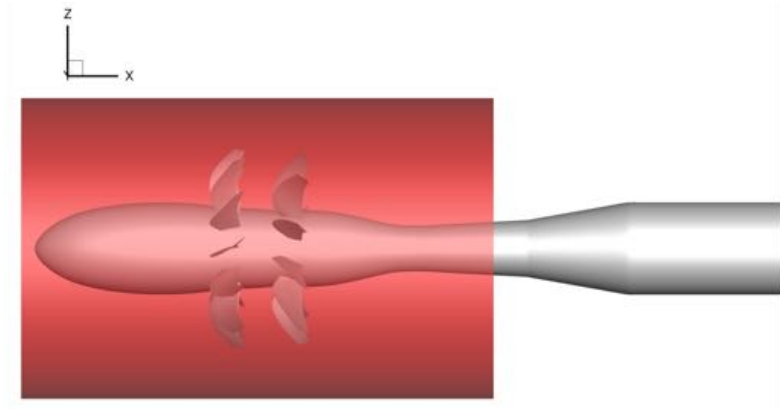

(b) Acoustic Surface 2

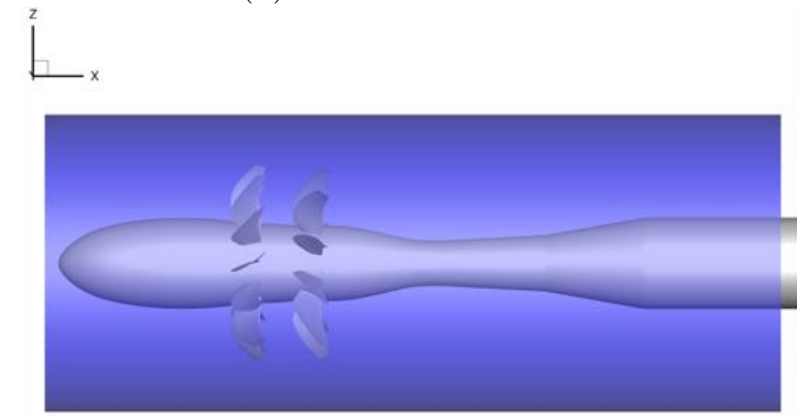

(c) Acoustic Surface 3

Figure 6. Permeable surfaces used for FW-H propagation. 


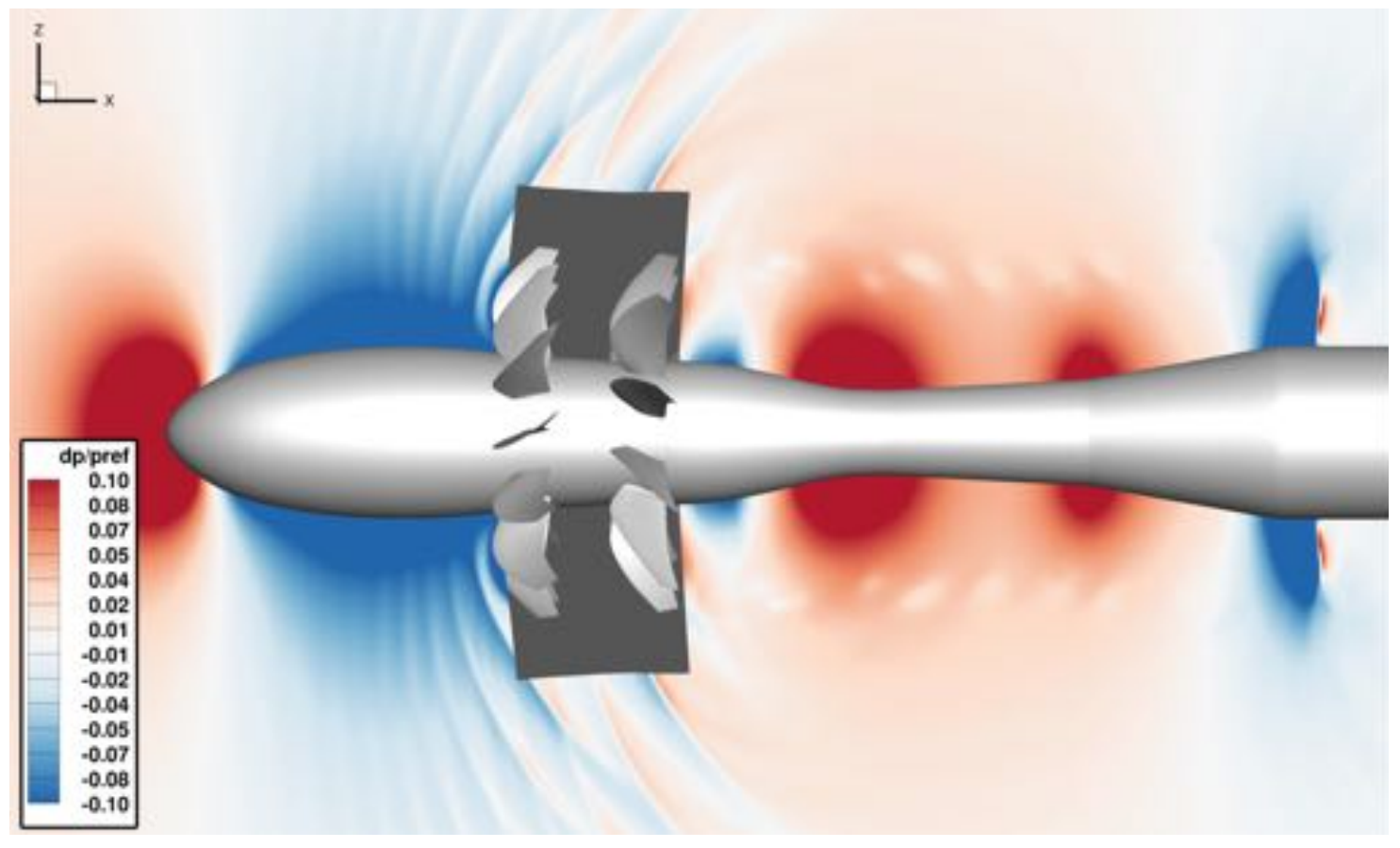

(a)

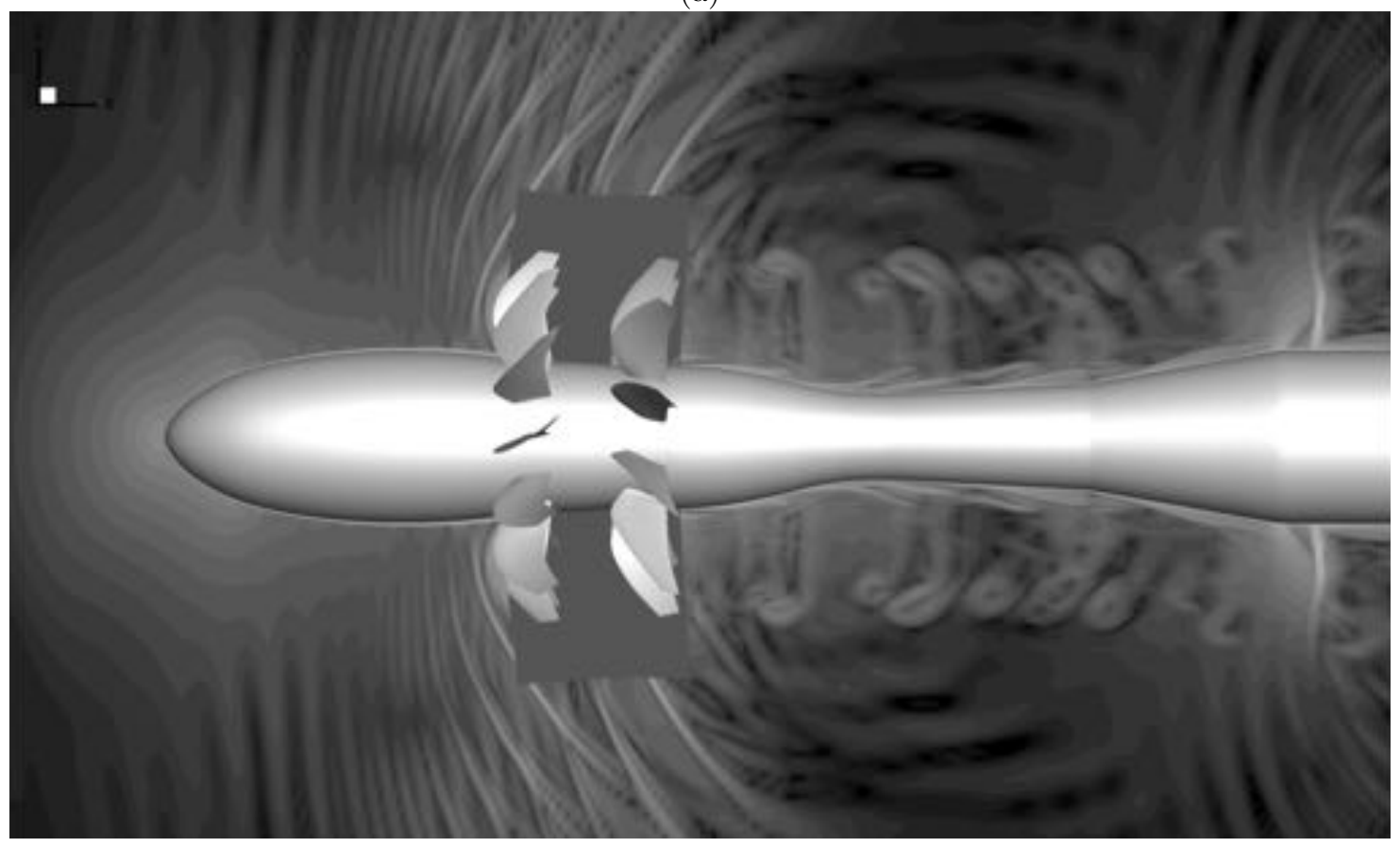

(b)

Figure 7. Contour plot of the (a) pressure and (b) magnitude of the density gradient on a slice through the centerline of the hub. 


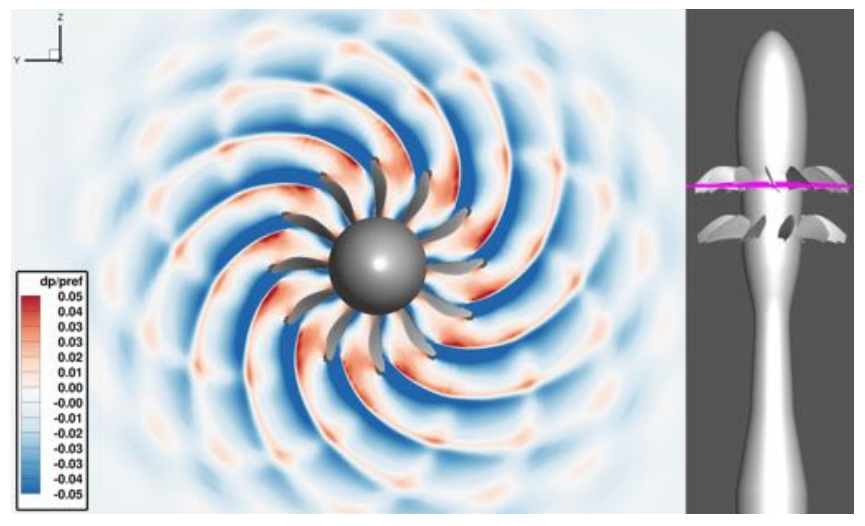

(a)

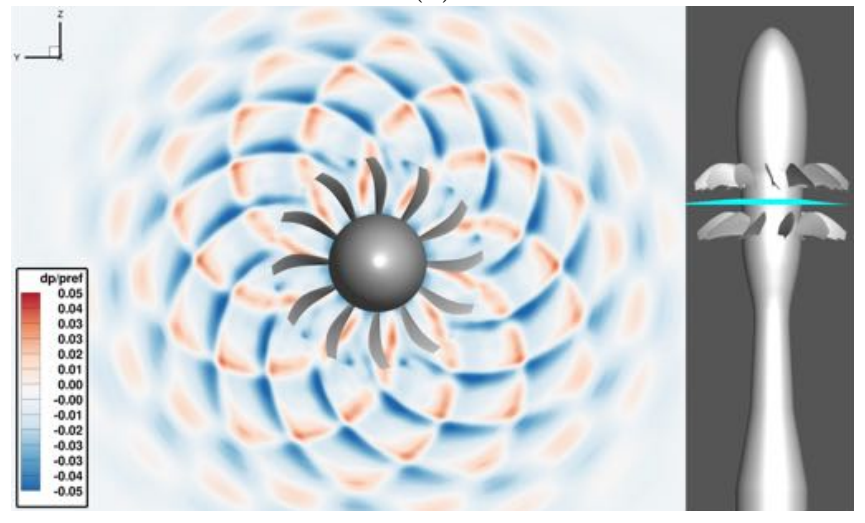

(b)

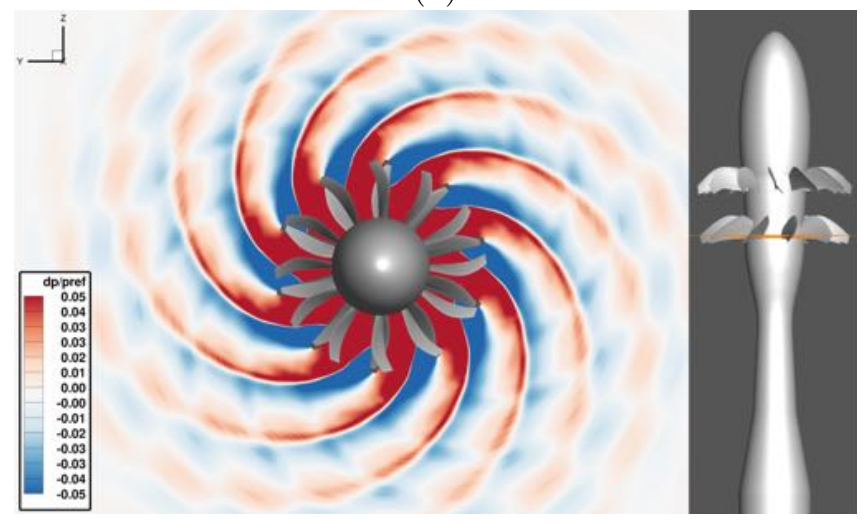

(c)

Figure 8. Contour plot of pressure on stream-wise slices of the grid (a) through the forward rotors, (b) between the two rotors, and (c) through the aft rotors 

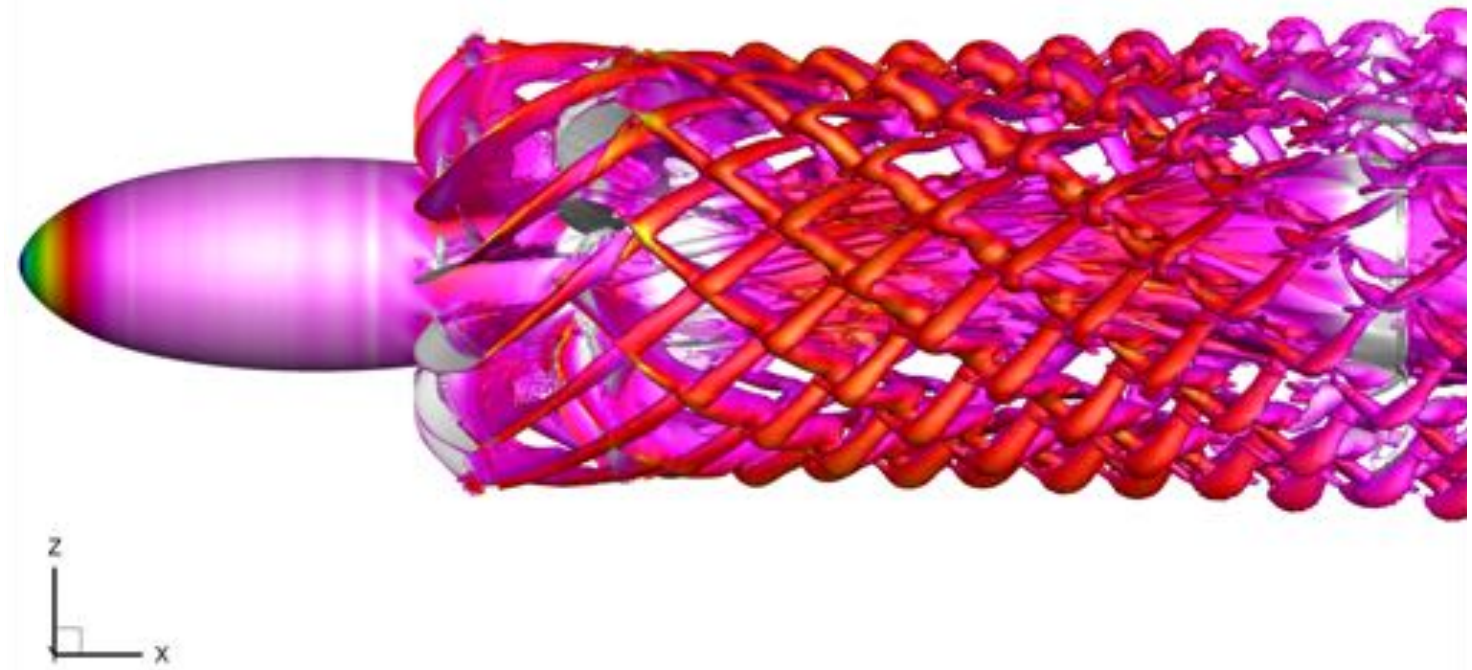

Figure 9. Iso-contour of vorticity magnitude at $2000(1 / \mathrm{s})$ colored my Mach number.

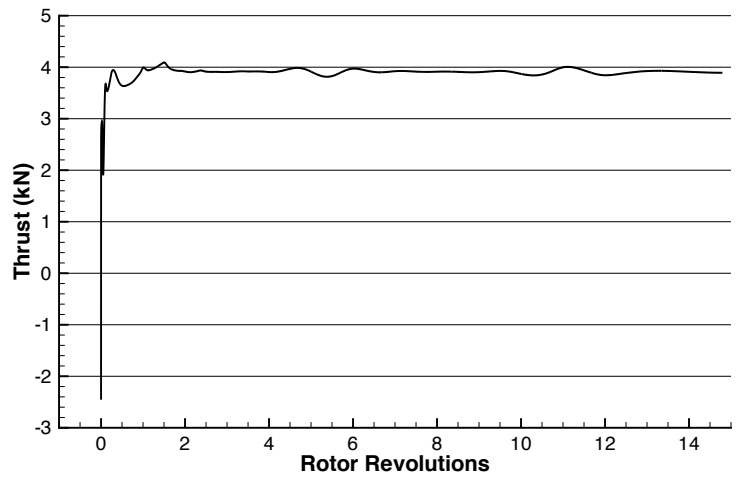

(a)

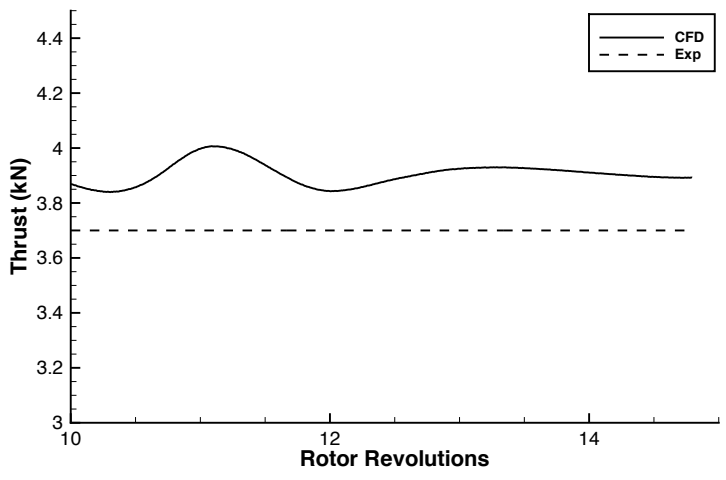

(b)

Figure 10. (a) Time evolution of the predicted thrust from the impulsively started CROR simulation. (b) Comparison of the predicted thrust over the last four rotor revolutions with the time-averaged thrust measured in the experiment. 


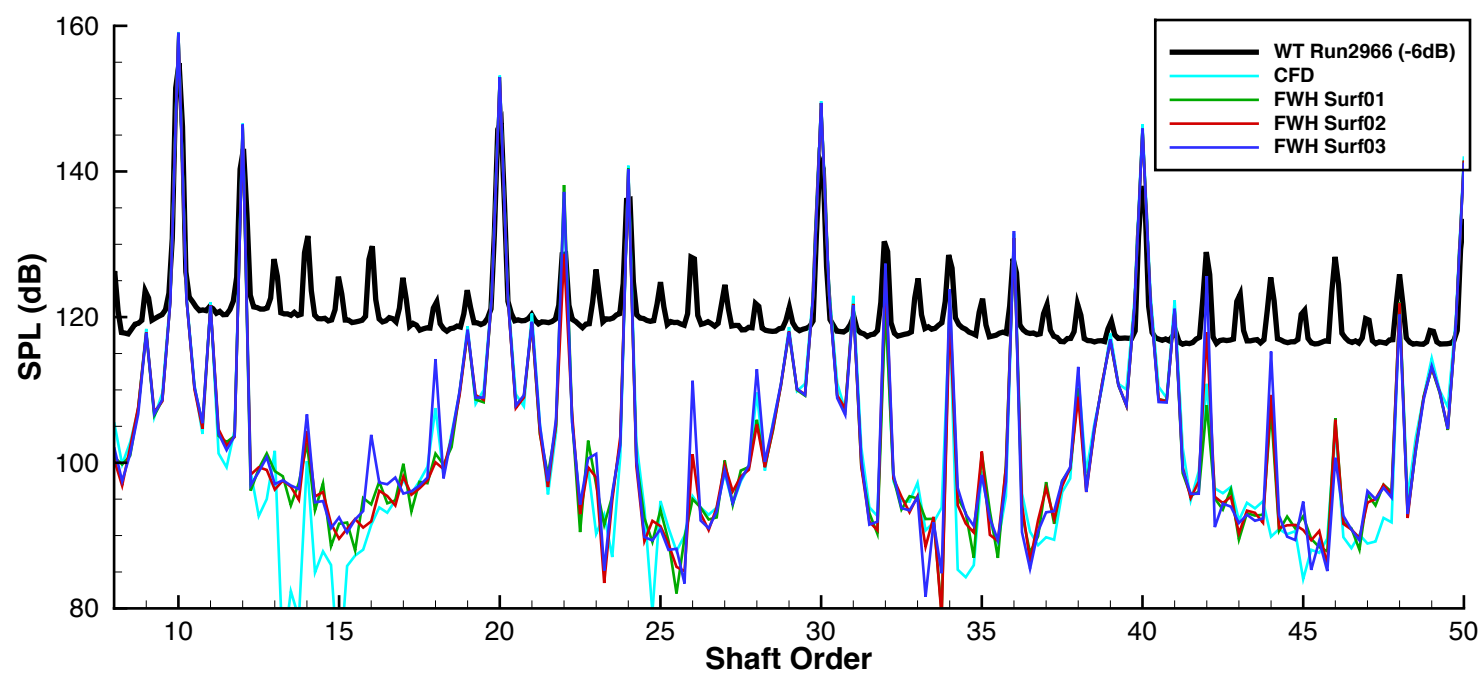

(a)

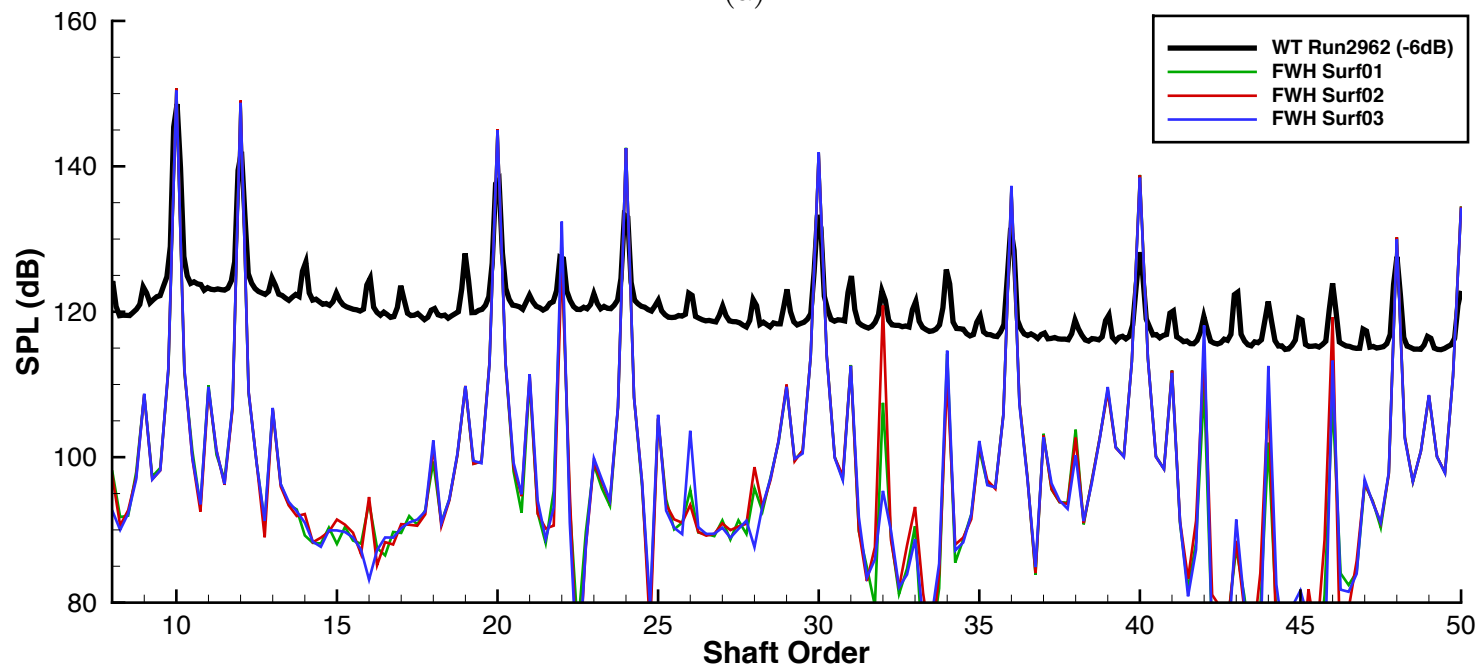

(b)

Figure 11. Sound pressure level (SPL) spectrum at Kulite 9 located above the aft rotor at a vertical distance of (a) $0.51 \mathrm{~m}$ and (b) $1.16 \mathrm{~m}$ from the centerline of the hub.

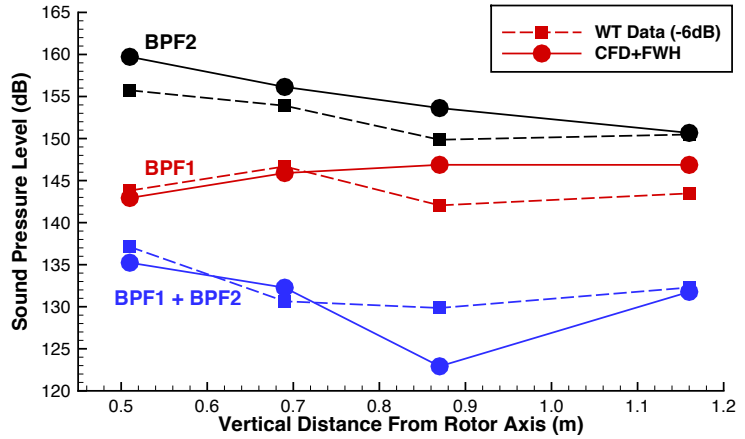

(a) Kulite 8

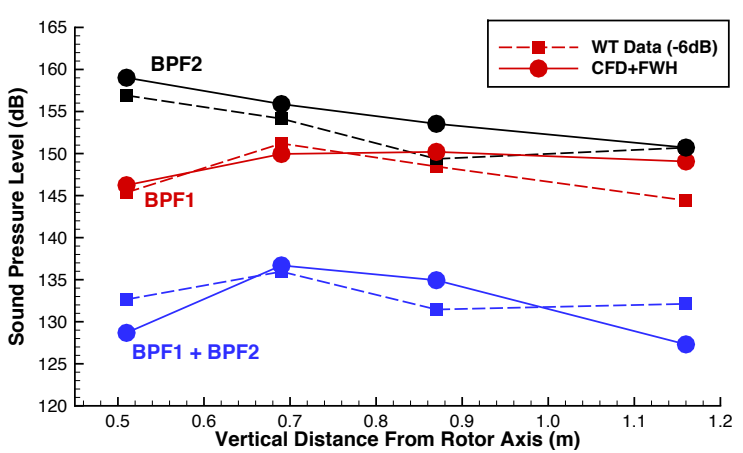

(b) Kulite 9

Figure 12. Comparison of tone SPL at (a) Kulite 8 and (b) Kulite 9 as a function of vertical distance from the hub centerline. Experimental measurements shown with dashed line and square symbols, predicted results shown with solid line and circles. A $6 \mathrm{~dB}$ reduction was applied to the measured data to remove the plate reflection effects. Permeable surface 2 was used for the FW-H acoustic propagation. 


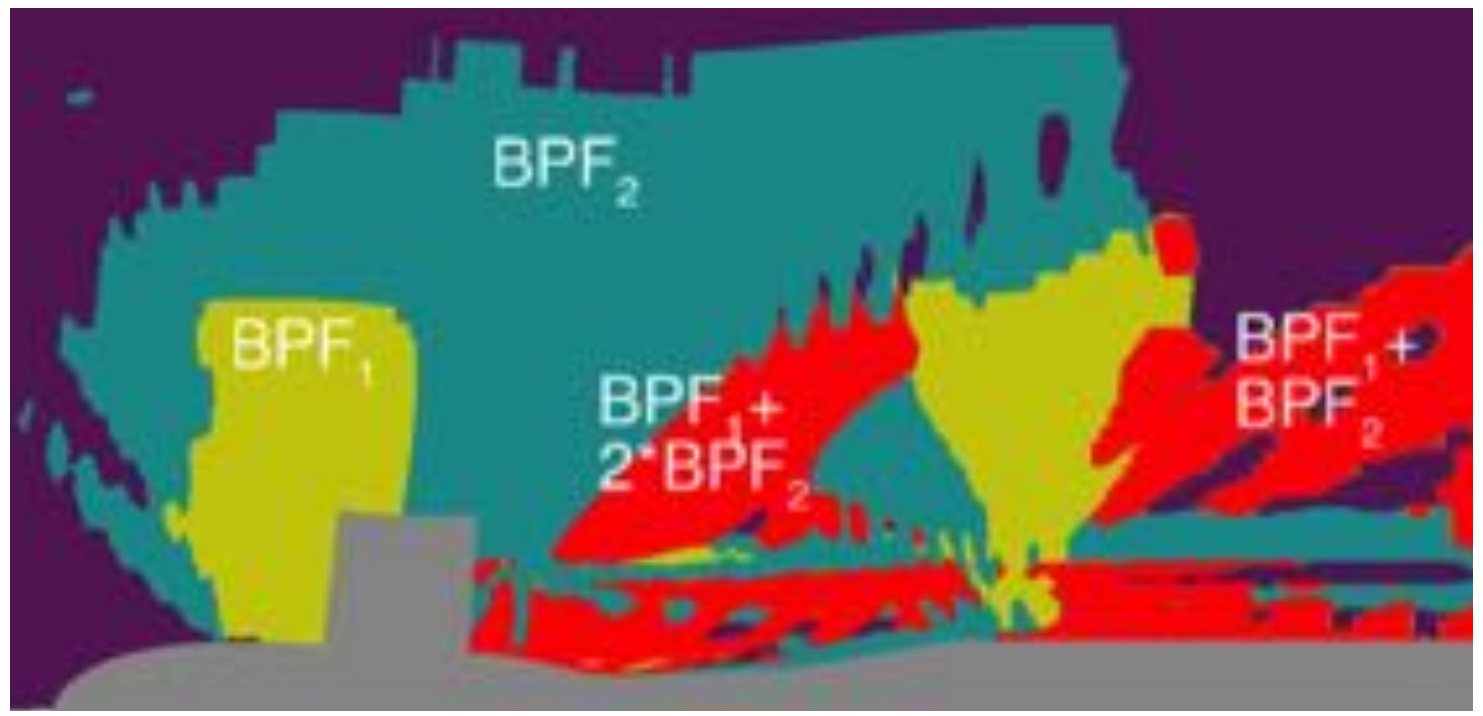

Figure 13. A frequency diagram plotting the frequency at which the largest amplitude in the narrow-band spectrum is obtained for each spatial location on a $y=0$ cutting plane through the hub.

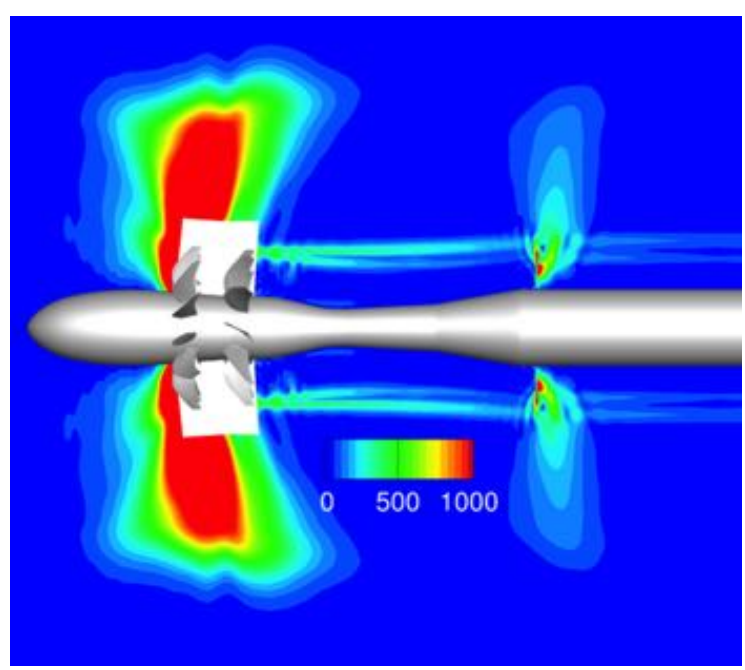

(a)

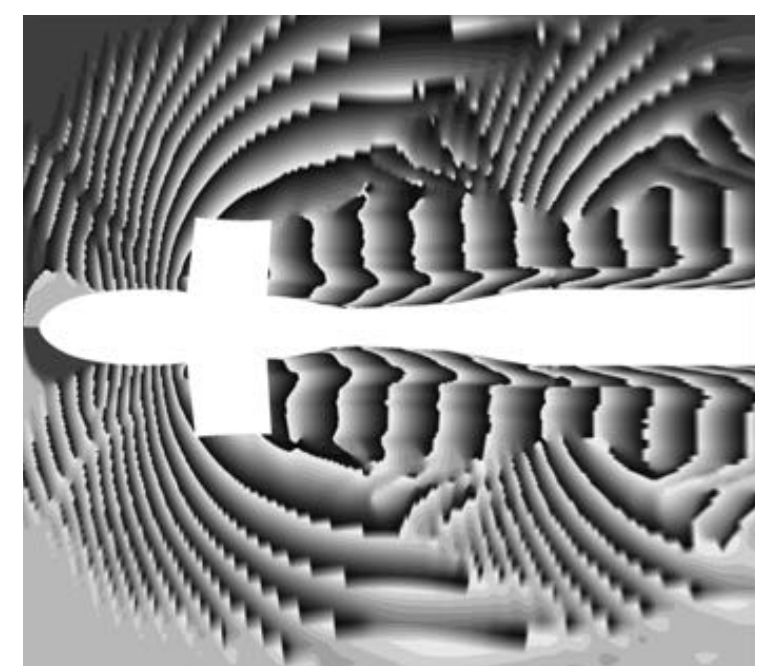

(b)

Figure 14. Plot of the (a) amplitude and (b) phase at $B P F_{1}$ on a $y=0$ cutting plane through the hub. 


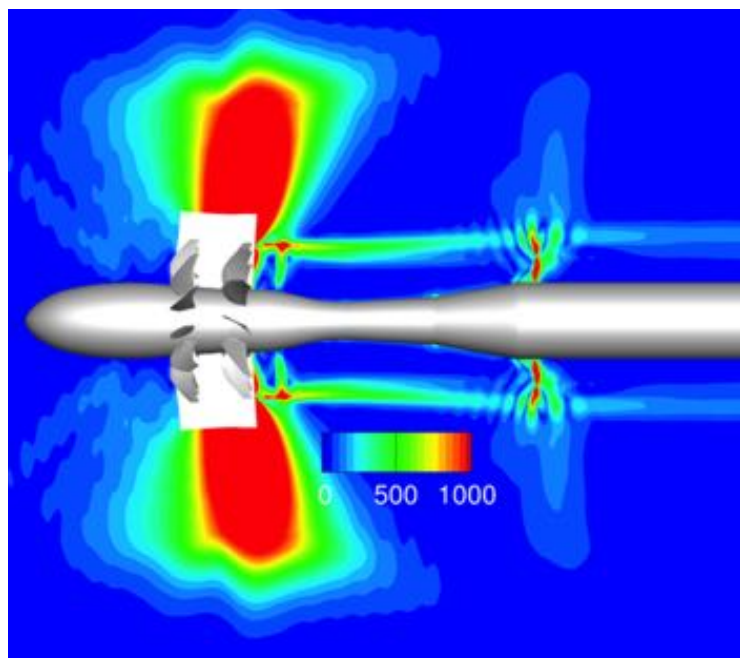

(a)

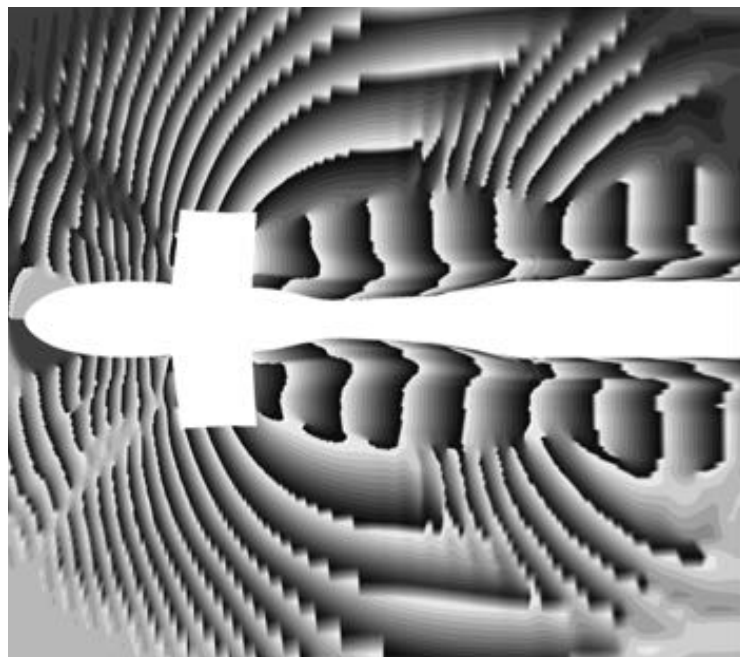

(b)

Figure 15. Plot of the (a) amplitude and (b) phase at $\mathrm{BPF}_{2}$ on a $\mathrm{y}=0$ cutting plane through the hub.

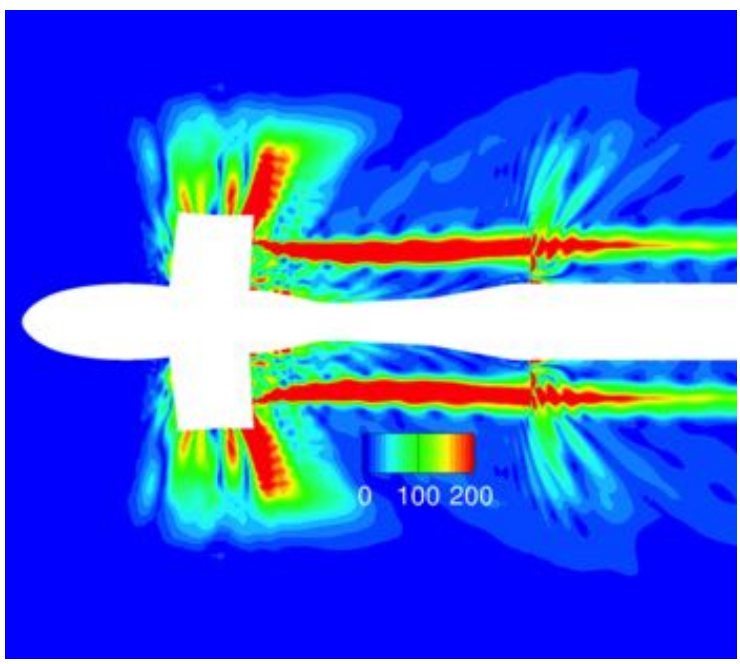

(a)

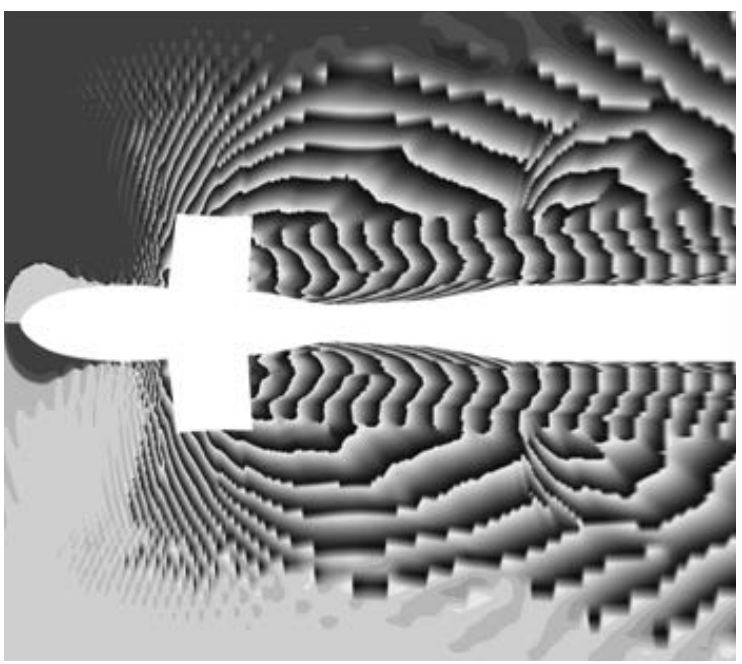

(b)

Figure 16. Plot of the (a) amplitude and (b) phase at $\mathrm{BPF}_{1}+\mathrm{BPF}_{2}$ on a $\mathrm{y}=0$ cutting plane through the hub. 


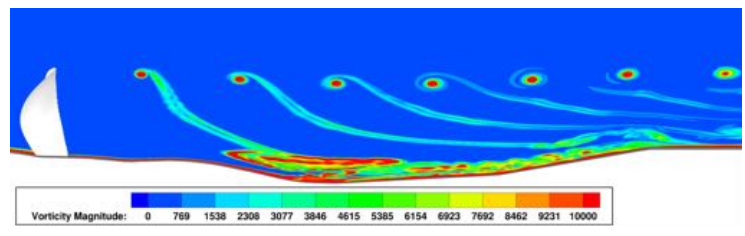

(a) 0.5 degrees

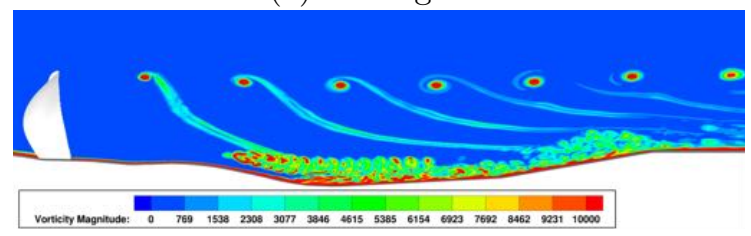

(c) 0.125 degrees

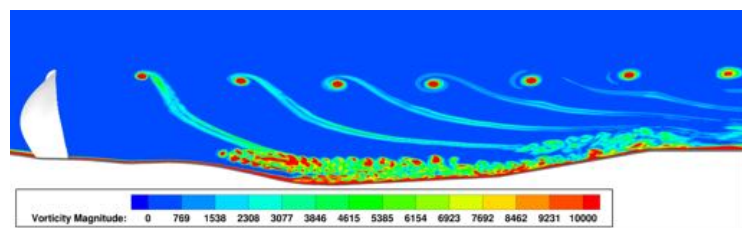

(e) 0.03125 degrees

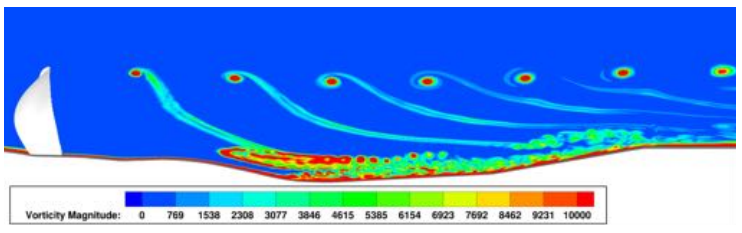

(b) 0.25 degrees

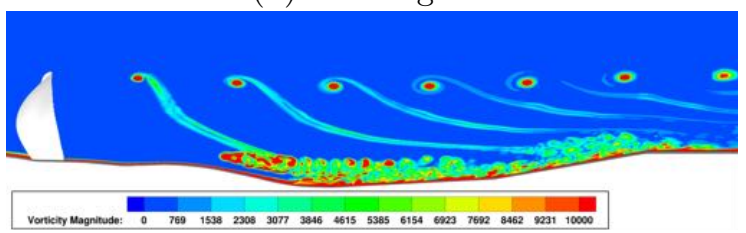

(d) 0.0625 degrees

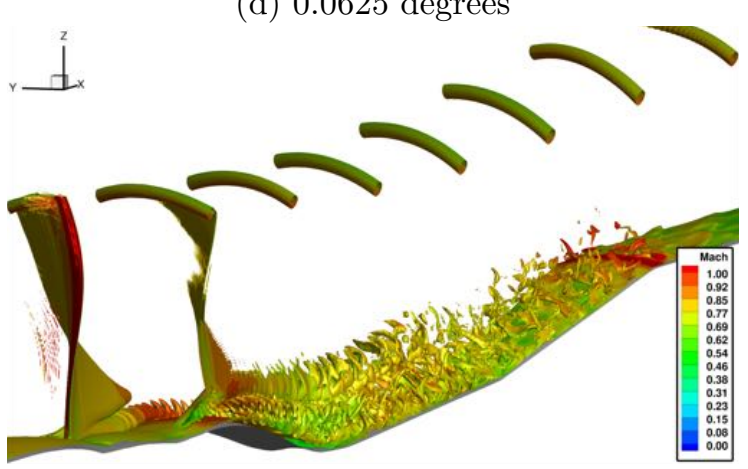

(f) Isometric View

Figure 17. Contour plot of instantaneous vorticity magnitude on a $y=0$ cutting plane through the hub for time-steps associated with (a) 0.5 to (e) 0.03125 degree rotation-per-step and (f) an isometric view of an iso-contour of vorticity magnitude colored by Mach number illustrating the separated flow region emanating from the blade-hub intersection.

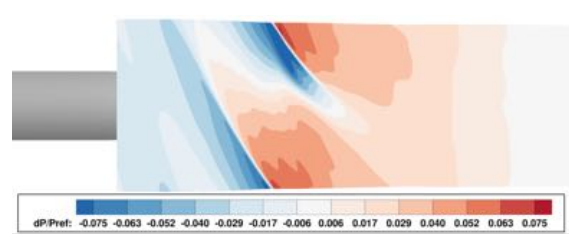

(a) 0.5 degrees

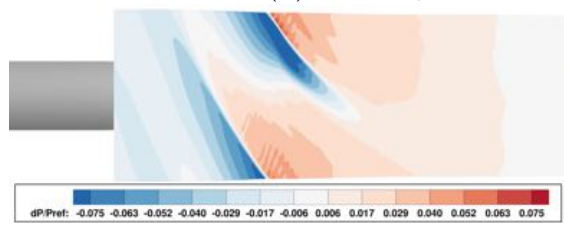

(c) 0.125 degrees

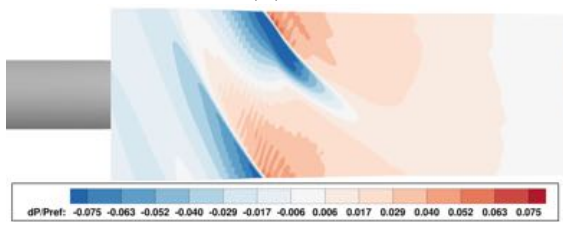

(e) 0.03125 degrees

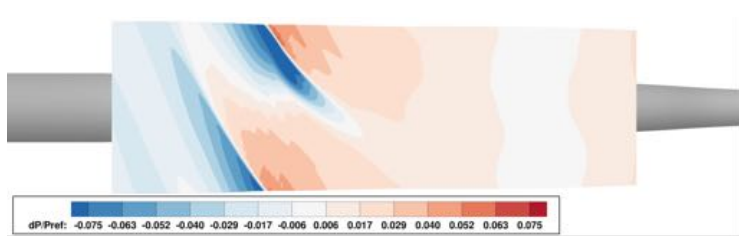

(b) 0.25 degrees

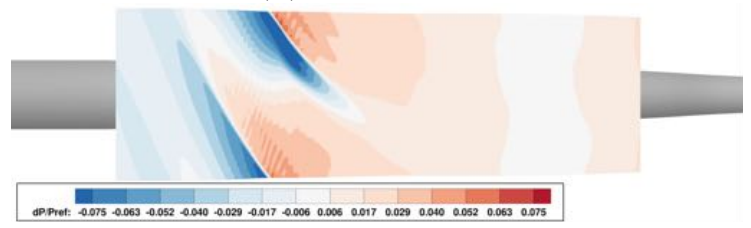

(d) 0.0625 degrees

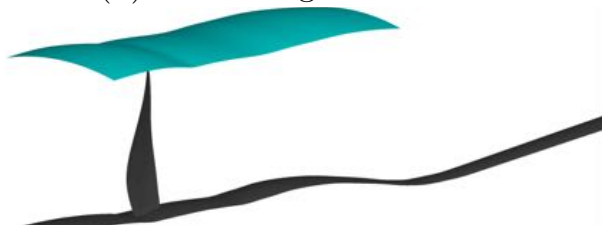

(f) Isometric View

Figure 18. Contour plot of instantaneous pressure on a constant radial grid surface above the forward blade for time-steps associated with (a) 0.5 to (e) 0.03125 degree rotation-per-step and (f) an isometric view of the surface. 


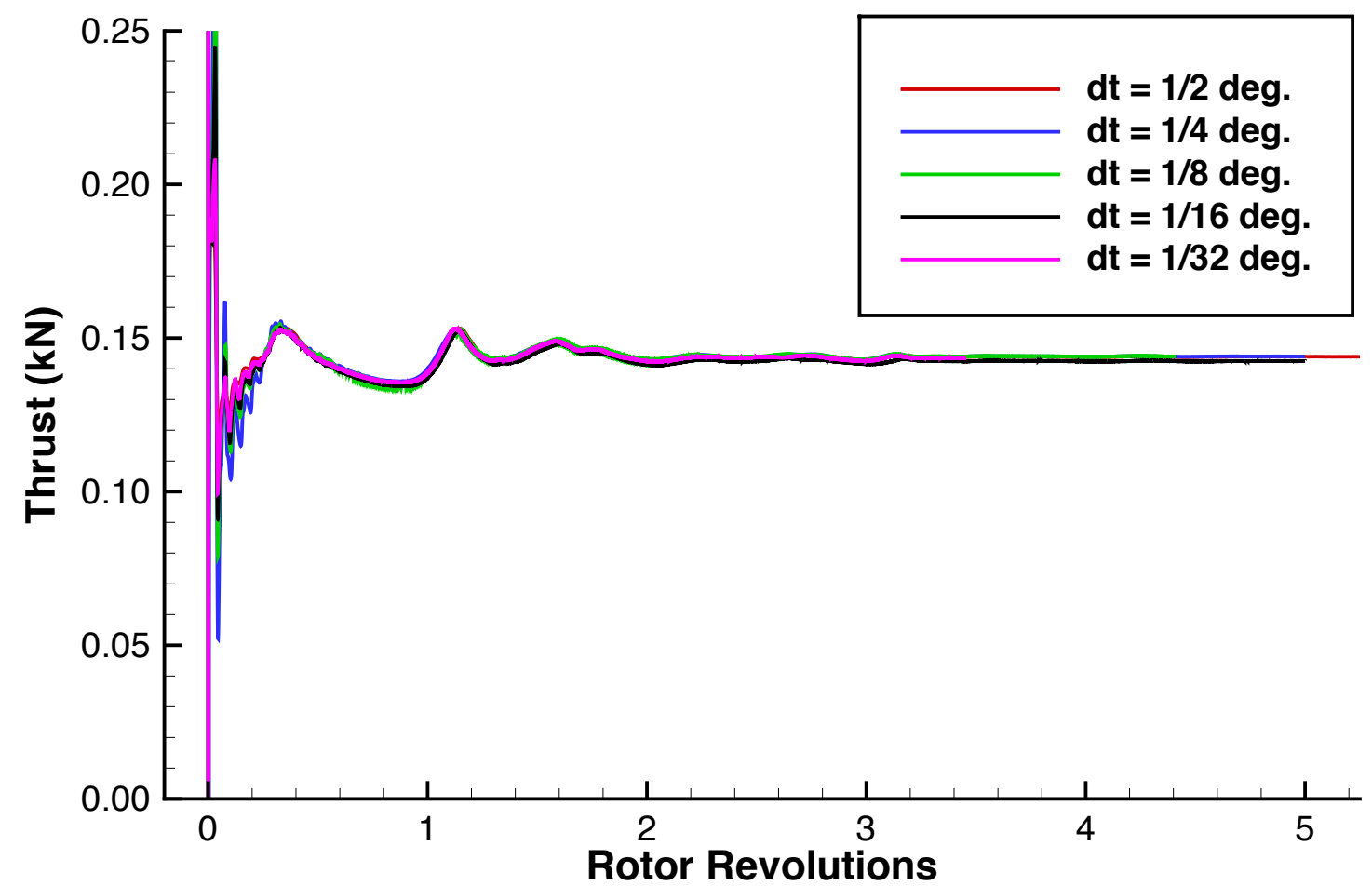

Figure 19. Time evolution of thrust on a single forward rotor using time-steps of $0.5,0.25,0.125,0.0625$, and 0.03125 degrees rotation-per-step. 